\title{
Safety and Tolerability of Antibody-Drug Conjugates in Cancer
}

\author{
Anna Wolska-Washer ${ }^{1} \cdot$ Tadeusz Robak $^{1}$
}

Published online: 16 January 2019

(c) The Author(s) 2019

\begin{abstract}
Antibody-drug conjugates are monoclonal antibodies attached to biologically active drugs through chemical linkers that deliver and release cytotoxic agents at the tumor site, reducing the likelihood of systemic exposure and therefore toxicity. Currently, there are about 110 ongoing studies implementing antibody-drug conjugates in the treatment of multiple human malignancies. Antibody-drug conjugates carry a feature of the specificity of a monoclonal antibody and the anti-neoplastic potential of a cytotoxin. The first antibody-drug conjugate was approved in 2001, and the field of antibody-drug conjugates has expanded since then with three more antibody-drug conjugates being added to the market. The complex structure of the antibody-drug conjugate poses a challenge in designing a clinically adequate molecule. Antibody-drug conjugates are usually well tolerated with some predictable adverse reactions, as well as new medical issues, that need careful approach. This review provides an outline of the current status of the efficacy and safety of antibody-drug conjugates in malignant diseases.
\end{abstract}

\section{Key Points}

Antibody-drug conjugates (ADCs) deliver and release cytotoxic agents at the tumor site with reduced systemic toxicity.

Recent progress in biotechnology has enabled more effective selection of cytotoxins and linkers, and has drastically improved the potency of ADCs in the clinical setting. However, there is still some concern about the immunogenicity, bystander or systemic toxicity, and rapid clearance, which should be overcome to better exploit the properties of ADCs.

The prioritization of novel ADCs entering clinical development for treating malignant disorders represents a promising treatment option for these frequently incurable diseases.

Part of a theme issue on "Safety of Novel Anticancer Therapies: Future Perspectives". Guest Editors: Rashmi R Shah, Giuseppe Curigliano.

Tadeusz Robak

robaktad@csk.umed.lodz.pl

1 Department of Hematology, Medical University of Lodz and Copernicus Memorial Hospital, Ul. Ciolkowskiego 2, 93-510 Lodz, Poland

\section{Introduction}

Antibody-drug conjugates (ADCs) selectively deliver large amounts of antitumor drugs to tumor tissue and show significant antitumor effects with a wide therapeutic window. Antibody-drug conjugates have emerged as a new modality in the treatment of various malignancies. They pose a unique feature of delivering and releasing cytotoxic agents at the tumor site or, even more precisely, within the target tumor cells. Thus, the outcome is the improved tumorto-normal tissue selectivity and specificity of the therapy. Currently, there are four ADCs approved by the US Food and Drug Administration (FDA) and the European Union: gemtuzumab ozogamicin, brentuximab vedotin, trastuzumab emtansine, and inotuzumab ozogamicin. In addition, several new promising agents are under development. This review provides an outline of the current status of the efficacy and safety of approved ADCs and novel investigational agents in malignant diseases. We also discuss the metabolic profile of approved agents as well as serious and life-threatening adverse reactions.

\section{Mechanism of Action}

The idea behind ADCs is the delivery of a potent cytotoxin to its target using a specific carrier. An ADC consists of a monoclonal antibody (mAb) linked to the cytotoxic drug via 
specialized linking molecules. The likelihood of systemic exposure and toxicity is greatly reduced, depending on the molecular target for the antibody part of the ADC and the linker's chemical stability in the circulation.

The key to obtain a medically relevant ADC is to optimize a few parameters including a suitable target, a powerful cytotoxic payload, an appropriate linker, and the technology to conjugate these at a proper antibody conjugation site. [1] This shows the degree of complexity in the field of ADC design and production. Currently used cytotoxic payloads derive from microorganisms (Micromonospora echinospora, Actinomycetes spp.), plants (Maytenus spp.), small-molecular-weight drugs (tubulin inhibitors, DNA cross-linking molecules), or even sea animals such as the sea hare (Dolabella auricularia), and have been further modified to exploit their potential. [2]

Antibody-drug conjugates use cleavable and non-cleavable linkers. [3] The first group includes pH-sensitive, glutathione-sensitive, lysosomal protease-sensitive, and $\beta$-glucuronidase-sensitive linkers. Their main disadvantage is the potential to release the payload in the blood stream, and therefore increase systemic toxicity and decrease effectiveness. Non-cleavable linkers remain stable in the blood stream and the whole ADC complex undergoes degradation within the target cell, leaving a payload-linker remnant with retained activity. Moreover, there is another major challenge that appears in any anti-cancer therapy, which is multi-drug resistance. It is either inherent or acquired, and may result from altered expression of the target antigen, different signaling pathways, or overexpression of efflux transporter proteins. [4] Virtually all used payloads may become potential substrates for P-glycoprotein (P-gp) and perhaps modifying the linking molecules of ADCs will effectively overcome this issue.

Finally, mAbs are generated by immortalized murine plasma cells. [1] They are genetically modified to produce a humanized $\mathrm{mAb}$ with a human $\mathrm{Fc}$ region and murine (or rat) Fab regions with complementarity-determining motifs. Therefore, the immunogenicity of such mAbs is greatly reduced, but not eliminated.

\section{Currently Approved Agents}

At the moment, there are four ADCs approved by the FDA and EU for the treatment of cancer (Table 1).

\subsection{Gemtuzumab Ozogamicin}

One of the first approved ADCs was gemtuzumab ozogamicin (GO; Mylotarg ${ }^{\circledR}$, Pfizer/Wyeth, USA) in 2001 under the brand name Mylotarg by Pfizer/Wyeth. It is built with a humanized anti-CD-33 immunoglobulin G4 mAb, a
$\mathrm{pH}$-sensitive hydrazone linker, and a calicheamicin derivative conjugated with the side-chain reactive lysine residues of mAbs. [5] Initially, the drug was indicated as monotherapy in elderly patients with acute myeloid leukemia (AML) with relapsed disease. The efficacy and safety of GO as a single agent have been evaluated in 142 patients in three single-arm open-label studies in patients with CD33-positive AML in first relapse. [6] The treatment involved two 9-mg/ $\mathrm{m}^{2}$ doses given on days 1 and 15 and a 28-day follow-up. The primary endpoint of the trials was complete remission (CR) and was achieved by $16 \%$ of all patients and $13 \%$ demonstrated CR with incomplete platelet recovery. In the trial with patients aged older than 60 years, both CR rate and $\mathrm{CR}$ with incomplete platelet recovery were $11 \%$ each. The $9-\mathrm{mg} / \mathrm{m}^{2}$ dose was chosen to saturate all CD33 sites regardless of leukemic burden. Median relapse-free survival for patients achieving any response was 6.8 months (range $0.33-24.8$ months), and median overall survival (OS) in the whole group was 5.9 months. Severe myelosuppression with prolonged agranulocytosis and thrombocytopenia was the most common (99\% patients) adverse reaction after GO. Nevertheless, GO was approved for use in patients ineligible for aggressive cytotoxic regimens, especially patients 60 years of age or older.

However, post-marketing studies revealed marked systemic toxicity and poor efficacy of GO. In a phase III SWOG S0106 randomized comparative trial, GO was used in combination with other chemotherapeutic agents (daunorubicin and cytosine arabinoside) vs. chemotherapy alone in 637 patients with first-line AML under the age of 61 years. [7] The study did not confirm the clinical benefit of GO in combination therapy, such as CR rate, disease-free survival, and OS. Moreover, the induction mortality rate due to toxicity was significantly higher in the combination arm, compared with chemotherapy alone (5.7\% vs. $1.4 \%$ of patients). The observed increased GO toxicity was probably caused by relatively high instability of the linker in the blood stream, combined with a high recommended dose. The drug was withdrawn from the market.

However, after the results of several clinical trials, GO returned in 2017 but at a lower recommended dose and with a different dosing schedule, which warrants the right benefitrisk ratio for the patients. Monotherapy with GO was studied in 294 patients: 237 with newly diagnosed AML and 57 patients with relapsed AML. The first study was a phase III trial that included patients above 60 years of age, who were not eligible or did not consent to receive intensive chemotherapy, and treatment options included GO or best supportive care. $[8,48]$ Gemtuzumab ozogamicin was administered at $6 \mathrm{mg} / \mathrm{m}^{2}$ on day 1 and $3 \mathrm{mg} / \mathrm{m}^{2}$ on day 8 in a 28-day regimen. The therapy was repeated up to eight monthly infusions at $2 \mathrm{mg} / \mathrm{m}^{2}$, provided the patient did not progress after the first infusion. Gemtuzumab ozogamicin had a statistically 
Table 1 Currently approved antibody-drug conjugates (ADCs) with indications issued by the US Food and Drug Administration (FDA) and European Medicines Agency (EMA)

\begin{tabular}{|c|c|c|c|c|}
\hline $\mathrm{ADC}$ & Brand name & Manufacturer & Indication & Year of approval FDA/EMA \\
\hline 1. Gemtuzumab ozogamicin & Mylotarg & Pfizer/Wyeth, USA & $\begin{array}{l}\text { FDA: in combination and as a single-agent } \\
\text { therapy in newly diagnosed CD33-positive } \\
\text { acute myeloid leukemia in adults; as a single } \\
\text { agent in relapsed or refractory CD33-positive } \\
\text { acute myeloid leukemia in adults and in pediat- } \\
\text { ric patients aged } 2 \text { y and older } \\
\text { EMA: in combination therapy with daunorubicin } \\
\text { and cytarabine for the treatment of patients } \\
\text { aged } \geq 15 \text { y with previously untreated, de novo } \\
\text { CD33-positive acute myeloid leukemia, except } \\
\text { acute promyelocytic leukemia }\end{array}$ & $\begin{array}{l}\text { 2001: discontinued } \\
\text { 2017/2018: re-approved }\end{array}$ \\
\hline 2. Brentuximab vedotin & Adcetris & Takeda Pharma & $\begin{array}{l}\text { FDA: adult patients with previously untreated } \\
\text { stage III or IV classical Hodgkin lymphoma, in } \\
\text { combination with chemotherapy (2018) } \\
\text { Classical Hodgkin lymphoma at high risk of } \\
\text { relapse or progression as post-auto-HSCT } \\
\text { consolidation } \\
\text { Classical Hodgkin lymphoma after failure of } \\
\text { auto-HSCT or after failure of at least two prior } \\
\text { multi-agent chemotherapy regimens in patients } \\
\text { who are not auto-HSCT candidates } \\
\text { sALCL after failure of at least one prior multi- } \\
\text { agent chemotherapy regimen } \\
\text { pcALCL or CD30-expressing MF who have } \\
\text { received prior systemic therapy (2017) } \\
\text { EMA: adult patients with classical Hodgkin } \\
\text { lymphoma after failure of auto-HSCT or } \\
\text { after failure of at least two prior multi-agent } \\
\text { chemotherapy regimens in patients who are not } \\
\text { auto-HSCT candidates } \\
\text { sALCL after failure of at least one prior multi- } \\
\text { agent chemotherapy regimen } \\
\text { pcALCL or CD30-expressing MF who have } \\
\text { received prior systemic therapy (2017) }\end{array}$ & $2011 / 2012$ \\
\hline 3. Trastuzumab emtansine & Kadcyla & Genentech, Roche & $\begin{array}{l}\text { FDA and EMA: adult patients with HER2-pos- } \\
\text { itive, unresectable locally advanced or meta- } \\
\text { static breast cancer who previously received } \\
\text { trastuzumab and a taxane. Patients should } \\
\text { have either received prior therapy for locally } \\
\text { advanced or metastatic disease, or developed } \\
\text { disease recurrence during or within } 6 \text { mo of } \\
\text { completing adjuvant therapy }\end{array}$ & 2013 \\
\hline 4. Inotuzumab ozogamicin & Besponsa & Pfizer/Wyeth & $\begin{array}{l}\text { FDA: in monotherapy in adults with relapsed or } \\
\text { refractory B-cell precursor ALL } \\
\text { EMA: in monotherapy in adults with relapsed } \\
\text { or refractory CD22-positive B-cell precursor } \\
\text { ALL. Adult patients with Philadelphia chromo- } \\
\text { some positive }(\mathrm{Ph}+) \mathrm{r} / \mathrm{r} \text { B-cell precursor ALL } \\
\text { should have not responded to treatment with at } \\
\text { least } 1 \text { tyrosine kinase inhibitor }\end{array}$ & 2017 \\
\hline
\end{tabular}

$A L L$ acute lymphoblastic leukemia, $H S C T$ hematopoietic stem cell transplantation, $M F$ mycosis fungoides, $p c A L C L$ primary cutaneous anaplastic large cell lymphoma, $s A L C L$ systemic anaplastic large cell lymphoma

significant advantage of OS over best supportive care with a median of 4.9 vs. 3.6 months, respectively. The 1-year OS was $24.3 \%$ with $\mathrm{GO}$ and $9.7 \%$ with best supportive care. The rates of adverse events (AEs) were comparable in both groups. The second group was studied in a phase II clinical trial and included elderly patients not eligible for intensive chemotherapy. [9] The treatment comprised either $3 \mathrm{mg} / \mathrm{m}^{2}$ of GO on days 1,3 , and 5 ( $\operatorname{arm~A)~or~} 6 \mathrm{mg} / \mathrm{m}^{2}$ of GO on day 1 and $3 \mathrm{mg} / \mathrm{m}^{2}$ on day 8 (arm B). The primary endpoint was the rate of disease non-progression, defined as either a 
response or maintaining stable disease after the treatment. The rate of disease non-progression was $38 \%$ in arm A, and $63 \%$ in arm B. The most common AEs were peripheral cytopenias. The all-cause early mortality rate was $14 \%$ in arm A and $11 \%$ in arm B. The day 1 plus eight dosing schedule met the statistical criteria to be selected as the preferred regimen for phase III comparison with best supportive care.

Combination therapy with GO was evaluated in a phase III ALFA-0701 trial with 278 patients with untreated de novo AML. [10] The control group received standard treatment with cytarabine and daunorubicin, and the experimental arm received additional GO at five fractionated doses of $3 \mathrm{mg} / \mathrm{m}^{2}$ on days 1,4 , and 7 during induction and day 1 of each of the two consolidation courses. Complete remission rate was higher in the experimental arm than in the control group ( 81 vs. $75 \%$, respectively). Event-free survival and OS at 2 years were $17.1 \%$ and $41.9 \%$ for the control arm, respectively, and $40.8 \%$ and $53.2 \%$ in the experimental arm, respectively. Common side effects included fever, nausea, infection, vomiting, and stomatitis. Severe side effects were low blood counts, liver damage including hepatic venoocclusive disease, infusion-related reactions (IRRs), and hemorrhage. The most common hematologic toxicity was persistent thrombocytopenia, which prevailed in the experimental arm (16\% vs. $3 \%$ respectively). The conclusion was that fractionated lower doses of GO provide safer delivery of higher cumulative doses that significantly improve patients' outcomes. Table 1 shows the current indications for GO by the FDA.

\subsection{Brentuximab Vedotin}

In 2011, brentuximab vedotin (BV, SGN-35; Adcetris, Seattle Genetics, USA) was introduced in USA by Seattle Genetics. The payload of this ADC is an auristatin derivative, $p$-aminobenzylcarbamate-monomethyl auristatin $\mathrm{E}$ (MMAE), conjugated to the anti-CD30-mAb cysteine residues. [11] Brentuximab vedotin utilizes a cathepsin B-sensitive linker that is degraded only upon internalization in tumor cells, but spared in systemic circulation owing to the higher $\mathrm{pH}$ and absence of cathepsin $\mathrm{B}$. Therefore, this linker is found to be superior to the hydrazone linker present in GO. Brentuximab vedotin also exerts the so-called bystander killing regardless of $\mathrm{CD} 30$ expression, as released MMAE easily diffuses through the cell membrane to the surrounding tissue. [11]

The maximum tolerated dose (MTD) of BV is $1.8 \mathrm{mg} / \mathrm{kg}$, administered intravenously every 3 weeks and was established in a phase I open-label multicenter dose-escalation study in a group of 45 patients with refractory CD30-positive hematologic malignancies. [12] Tumor regression was observed in 36 of 42 patients who could be evaluated $(86 \%)$. Objective responses were observed in 17 patients,
11 of whom had CRs. The median duration of response was 9.7 months. The most common AEs were peripheral neuropathy (PN) (66\%), fatigue (52\%), nausea (50\%), and diarrhea (32\%). The FDA approval was based on the results of two phase II clinical trials. The first one involved 102 patients with refractory or relapsed Hodgkin lymphoma (HL), who achieved objective responses in $75 \%$ of cases, with $34 \%$ CRs. [13] Overall median OS was 40.5 months, and it was not reached in the group with CRs. The second trial included 58 patients with relapsed or refractory anaplastic large cell lymphoma. The trial showed objective responses in $86 \%$ of cases with 53\% CRs. [14] The treatment with BV was complicated primarily by mild-to-moderate toxic effects, including diarrhea, nausea, neutropenia, thrombocytopenia, upper respiratory infection, PN, fatigue, and pyrexia. The most common grade 3 or 4 AEs were neutropenia $(21 \%)$, PN $(10 \%)$, thrombocytopenia (14\%), and anemia (7\%).

$\mathrm{An}$ addition to the initial indication for BV by the FDA is the treatment for patients with HL after autologous stem cell transplantation (ASCT) who are at high risk of relapse or progression after the procedure, and therapy should commence following recovery after ASCT (Table 1). The variation in application was supported by data from a randomized double-blind placebo-controlled phase III study named AETHERA, comparing BV and best supportive care $(n=165)$ with placebo and best supportive care $(n=164)$. [15] Inclusion criteria involved patients at high risk of residual HL post-ASCT. The patients could receive up to 16 cycles of BV monotherapy. The median PFS in the BV group was 42.9 months and in the placebo group it was 24.1 months. Median OS was not reached in either group. In both subgroups, similar numbers of patients were off study treatment at the time of the analysis. However, the main reason for patients being off treatment was different. In the BV subgroup, the main reason was an $\mathrm{AE}(n=54$, vs. $n=10$ in the placebo arm). In the placebo arm, the main reason was progressive disease ( $n=69$, vs. $n=24$ in the BV arm). The patients who progressed or relapsed in the placebo group and received BV still experienced prolonged PFS in $15-20 \%$ of cases.

Finally, BV is indicated for the treatment of adult patients with relapsed or refractory CD30 + cutaneous T-cell lymphoma. Its efficacy and safety as a single agent was evaluated in a phase III open-label randomized ALCANZA study in 128 patients with relapsed or refractory CD30-positive cutaneous T-cell lymphoma, including mycosis fungoides or primary cutaneous anaplastic large cell lymphoma. [16] Patients were randomized to receive either BV or either methotrexate or bexarotene. The median number of cycles was 12 in the BV arm. The results showed a consistent trend towards benefit for patients in the BV arm compared with patients who received physician's choice therapy. The median PFS was 16.7 in the BV group vs. 3.5 months in the 
physician's choice arm. Grade 3-4 AEs were reported in $41 \%$ of patients in the BV group and $47 \%$ of patients in the physician's choice group. Peripheral neuropathy of grade 2 and 3 was observed in $67 \%$ of patients receiving BV and in $6 \%$ of in the control group. One of the four on-treatment deaths was reported by the investigator to be BV related.

In March 2018, the FDA approved BV in the treatment of adult patients with previously untreated stage III or IV classical HL in combination with chemotherapy (doxorubicin, vinblastine, and dacarbazine). The approval was based on a randomized trial, ECHELON-1, which involved 1334 patients receiving either BV plus doxorubicin, vinblastine, and dacarbazine (BV + AVD) or bleomycin plus AVD (ABVD). [17] Patients were randomized to receive up to six cycles of BV + AVD or ABVD on days 1 and 15 of each 28-day cycle. The recommended dose of BV in combination with chemotherapy for de novo advanced HL was established at $1.2 \mathrm{mg} / \mathrm{kg}$ intravenously every 2 weeks for 12 doses. The estimated median PFS was not reached in either arm, with a median follow-up time of 24.9 months.

Neutropenia occurred in $58 \%$ of the patients receiving BV + AVD and in $45 \%$ of those receiving ABVD. Peripheral neuropathy was reported in $67 \%$ of patients in the BV + AVD group and in $43 \%$ of patients in the ABVD group. Pulmonary toxicity of grade 3 or higher was observed in less than $1 \%$ of patients receiving $\mathrm{BV}+\mathrm{AVD}$ and in $3 \%$ of those receiving ABVD. Among the deaths that occurred during treatment, seven of nine in the BV + AVD group were associated with neutropenia and 11 of 13 in the ABVD group were associated with pulmonary-related toxicity.

The most common AEs affecting at least $20 \%$ of patients treated with $\mathrm{BV}$ across all clinical trials were neutropenia, anemia, peripheral sensory neuropathy, nausea, fatigue, constipation, diarrhea, vomiting, and pyrexia. Primary granulocyte-colony stimulating factor prophylaxis is recommended with BV plus chemotherapy for the frontline treatment of advanced HL. Brentuximab vedotin has not yet been approved for upfront therapy in HL in the European Union.

\subsection{Trastuzumab Emtansine}

Trastuzumab emtansine (T-DM1; Kadcyla ${ }^{\circledR}$, Roche, Switzerland) is another potent ADC approved by the FDA and the EU in 2013. It is also known as ado-trastuzumab emtansine to avoid dispensing errors with trastuzumab. It is indicated for patients with HER2-positive, unresectable, locally advanced or metastatic breast cancer who previously received trastuzumab and a taxane, separately or in combination. [18] A recombinant humanized immunoglobulin G1 mAb trastuzumab targets the HER2 receptor, which is overexpressed on cancer cells and the warhead delivers the maytansinoid derivative DM1. The payload is released as a drug-linker residue upon proteolytic degradation of the
$\mathrm{mAb}$ in lysosomes. The MTD was established in a phase I clinical trial in 24 patients at $3.6 \mathrm{mg} / \mathrm{kg}$ given intravenously once every 3 weeks. [19] T-DM1 was associated with mild reversible toxicity and substantial clinical activity in a heavily pretreated population. The most common AEs included severe thrombocytopenia (54.2\% of patients), elevated transaminases $(41.7 \%)$, fatigue $(37.5 \%)$, anemia $(29.2 \%)$, and nausea (25.0\%). These AEs were generally grade 1-2 and reversible. [19]

The approval of T-DM1 was based on several clinical trials that proved its efficacy and safety. A phase III randomized open-label clinical study EMILIA was conducted in 991 patients with unresectable or metastatic breast cancer comparing T-DM1 with lapatinib plus capecitabine. [20] T-DM1 significantly delayed time to symptom progression by 7.1 months compared with 4.6 months in the control arm. The median PFS for the experimental arm was 9.6 months, compared with 6.4 months for the lapatinib plus capecitabine group. The benefit of T-DM1 was seen across the majority of evaluated subgroups. The median OS was 29.9 months in the T-DM1 arm, compared with 25.9 months in the lapatinib plus capecitabine arm. Two hundred and eleven (43.1\%) patients experienced grade $\geq 3$ AEs in the T-DM1 arm, compared with 289 (59.2\%) patients in the lapatinib plus capecitabine arm. The most frequently reported grade 3 or worse AEs in the T-DM1 group were thrombocytopenia (14\%), increased aspartate aminotransferase levels (5\%), and anemia (4\%). Thirty-two patients (6.5\%) discontinued T-DM1 because of an AE, compared with 41 patients (8.4\%) who discontinued lapatinib, and 51 patients $(10.5 \%)$ who discontinued capecitabine.

Thrombocytopenia and elevated transaminases were the most common AEs leading to T-DM1 withdrawal. Adverse events leading to dose reductions were reported in $16.3 \%$ of patients treated with T-DM1. The most frequent AEs leading to dose reduction of T-DM1 included thrombocytopenia, increased transaminases, and PN. Dose delays due to neutropenia, thrombocytopenia, leukopenia, fatigue, increased transaminases, and pyrexia occurred in $23.7 \%$ of patients.

A phase II randomized open-label study evaluated the efficacy of T-DM1 vs. trastuzumab plus docetaxel in patients with HER2-positive metastatic breast cancer who had not received prior chemotherapy for metastatic disease. [21] A total of 137 patients were treated in the study. The median PFS was 14.2 months in the T-DM1 arm and 9.2 months in the trastuzumab plus docetaxel arm. The median duration of response was not reached with T-DM1 compared with 9.5 months in the control arm. T-DM1 had fewer grade $\geq 3$ AEs than the control arm (46.4\% vs. $90.9 \%)$, AEs leading to treatment discontinuation ( $7.2 \%$ vs. $34.8 \%$ ), and serious AEs (20.3\% vs. $25.8 \%)$.

Another phase II single-arm study evaluated T-DM1 in patients with HER2-positive incurable, locally advanced 
or metastatic breast cancer. [22] All patients had previously received HER2-directed therapies (trastuzumab and lapatinib), and chemotherapy (anthracycline, taxane, and capecitabine). One hundred and ten patients were administered T-DM1 until disease progression or unacceptable toxicity. The objective response rate (ORR) was $32.7 \%$. The most common AEs were grade 1-2, and the most frequent grade $\geq 3$ AEs were thrombocytopenia (9.1\%), fatigue $(4.5 \%)$, and cellulitis (3.6\%).

Finally, a phase III randomized open-label trial of T-DM1 vs. physician's choice- TH3RESA demonstrated a significantly longer PFS for T-DM1 in previously treated patients with HER2-positive advanced breast cancer. [23] Moreover, the final analysis proved OS was significantly longer with T-DM1 vs. treatment of physician's choice (median 22.7 months vs. 15.8 months). Forty-seven percent of 198 patients in the physician's choice group had crossed over to the T-DM1 arm. The most common grade 3 or worse AEs in the T-DM1 arm were thrombocytopenia and hemorrhage of any type. [23, 24] The obtained data confirm the role of T-DM1 in the treatment of patients with previously treated HER2-positive advanced breast cancer, even after multiple lines of previous therapy.

\subsection{Inotuzumab Ozogamicin}

Inotuzumab ozogamicin (IO, CMC-544; Besponsa ${ }^{\circledR}$, Pfizer/ Wyeth) is the fourth ADC available on the market since 2017, both in the USA and Europe. It is a recombinant humanized anti-CD22 immunoglobulin G4 kappa mAb linked via an acid-sensitive hydrazone linker to a potent calicheamicin derivative. [3] It is currently indicated as monotherapy for the treatment of adult patients with relapsed or refractory CD22-positive B-cell acute lymphoblastic leukemia. [25] The recommended dose is $1.8 \mathrm{mg} / \mathrm{m}^{2}$ per cycle, administered as three divided doses on day $1\left(0.8 \mathrm{mg} / \mathrm{m}^{2}\right)$, day $8\left(0.5 \mathrm{mg} / \mathrm{m}^{2}\right)$, and day $15\left(0.5 \mathrm{mg} / \mathrm{m}^{2}\right)$. The treatment should include two cycles if the patient proceeds to hematopoietic stem cell transplantation (HSCT) or up to six cycles as the only therapy. The safety, antitumor activity, pharmacokinetics, and pharmacodynamics of IO were assessed in a phase I/II study for CD22-positive relapsed or refractory acute lymphoblastic leukemia. [26] In the phase I trial, a total of 37 patients received IO at $1.2,1.6$, or $1.8 \mathrm{mg} / \mathrm{m}^{2}$ per cycle on days 1,8 , and 15 over a 28 -day cycle (six or fewer cycles).

The recommended phase II dose was set at $1.8 \mathrm{mg} / \mathrm{m}^{2} /$ cycle, administered at a dose of $0.8 \mathrm{mg} / \mathrm{m}^{2}$ on day 1 and $0.5 \mathrm{mg} / \mathrm{m}^{2}$ on days 8 and 15 of a 28 -day cycle. Thirtyfive patients were treated in phase II. Cytopenias were the most common treatment-related toxicities. There were four reported cases of veno-occlusive disease/sinusoidal obstruction syndrome (VOD/SOS), including one fatal case. Two
VOD/SOS events occurred during treatment without HSCT; two patients experienced VOD/SOS after transplant. Complete remission and $\mathrm{CR}$ with incomplete hematologic recovery was reported in $68 \%$ of patients, and $84 \%$ of patients achieved minimal residual disease negativity. Median PFS was 3.9 months. Median OS was 7.4 months for all treated patients.

The approval of IO was based on a global phase III randomized open-label INO-VATE ALL study, assessing the clinical activity and safety of a single-agent InO compared with standard intensive chemotherapy in adults with relapsed or refractory $\mathrm{B}$-cell $\mathrm{pH}$-positive or $\mathrm{pH}$-negative acute lymphoblastic leukemia, given as the first or second salvage treatment. [27] Patients in the standard therapy arm received (per investigator's choice) either FLAG (fludarabine, cytarabine, granulocyte-colony stimulating factor), cytarabine plus mitoxantrone, or high-dose cytarabine. One hundred and thirty-nine patients received IO. Patients in the IO arm received a median of three (range 1-6) treatment cycles vs. 1 (range 1-4) cycle in the standard arm. The CR and $\mathrm{CR}$ with incomplete hematologic recovery rate was significantly higher with $\mathrm{InO} 80.7 \%$ vs. $33.3 \%$.

Significantly more patients proceeded to stem cell transplant directly after treatment with IO vs. standard therapy (41\% vs. $11 \%$ ). The most common AEs included cytopenias (thrombocytopenia, febrile neutropenia) and were slightly less prevalent in the IO arm. Other non-hematologic AEs were nausea, headache, and pyrexia, and were less common than in the standard care arm. Inotuzumab ozogamicin caused more hepatic toxicities, including elevated aspartate aminotransferase ( $20 \%$ vs. $10 \%)$, hyperbilirubinemia ( $15 \%$ vs. $10 \%)$, and increased alanine aminotransferase (14\% vs. $11 \%)$. Treatment-emergent hepatotoxicities were more frequent in the IO group (51\% vs. 34\%). [28] Thirteen percent of patients experienced VOD/SOS during treatment or after the treatment and the following HSCT, compared with less than $1 \%$ in the control group. Three percent of patients developed VOD/SOS after IO, compared with none in the control group. The estimated probability of survival was $38.9 \%$ in the IO group vs. $28.7 \%$ in the standard care group. The cases of VOD/SOS were reported for up to 2 years from randomization.

\section{Metabolic Profile}

Antibody-drug conjugates comprise three elements: a mAb, a linker, and a cytotoxic drug. Each of these components undergoes distinct metabolic changes. [1,29] Some linkers are processed intracellularly to release the cytotoxic drug, by a reduction in disulfide bonds mediated by glutathione in the cytoplasm, exposure to acidic conditions in the lysosome, or cleavage by specific proteases. The so-called non-cleavable 
linkers, that maintain better stability in systemic circulation, rely on degradation of the $\mathrm{mAb}$ to release their payload. Antibody-drug conjugates are not a homogenous solution. [29] After the administration, ADCs are being distributed and metabolized. The ADC is expected to be catabolized as a protein with component amino acids recycled or eliminated. Owing to their complex structure, bioanalysis should include the conjugated antibody, the total antibody, the antibody-conjugated drug, the unconjugated drug, and possibly its metabolites including or not part of the linker. [30]

Another vital component of the ADC therapeutic system are anti-drug antibodies (ADAs). All these parameters might impact the exposure-response effect relationship. Based on a population-pharmacokinetic (PK) analysis, following intravenous administration, the central volume of distribution of all ADCs equals approximately that of plasma volume, owing to the big molecular weight of the conjugates. [28]

\subsection{Gemtuzumab Ozogamicin}

To evaluate the pharmacokinetics of GO, it is necessary to measure PK characteristics of the whole molecule, as well as total and unconjugated calicheamicin metabolites. However, low systemic levels of unconjugated calicheamicin [a predicted mean maximum plasma concentration $\left(C_{\max }\right)$ of $1.5 \mathrm{ng} / \mathrm{mL}$ following the third dose] limited the determination of its PK parameters. [5, 31, 32] It was observed that the predicted total area under the curve (AUC) during the treatment was $25 \%$ and the $C_{\max }$ of GO is $24 \%$ of the values for the original $9-\mathrm{mg} / \mathrm{m} 2$ dosing regimen. With doses of $3 \mathrm{mg} / \mathrm{m}^{2}$ on days 1,4 , and 7 , the $C_{\max }$ of GO was predicted to be $0.38 \mathrm{mg} / \mathrm{L}$ after the first dose, and $0.63 \mathrm{mg} / \mathrm{L}$ after the third dose. The total volume of distribution was estimated at approximately $25 \mathrm{~L}$.

Gemtuzumab ozogamicin releases the calicheamicin derivative ( $N$-acetyl-gamma-calicheamicin-dimethyl hydrazide) via hydrolysis. Subsequently, the derivative undergoes non-enzymatic reduction of the disulfide bonds within the molecule. Therefore, there is little or no effect on exposure if GO is co-administered with cytochrome P450 inducers, inhibitors, or uridine diphosphate glucuronosyltransferase (UGT) drug-metabolizing enzymes. The metabolites exert little anti-neoplastic activity. The predicted clearance of GO from plasma was estimated at $3 \mathrm{~L} / \mathrm{h}$ after the first dose, and $0.3 \mathrm{~L} / \mathrm{h}$ thereafter. The terminal plasma half-life of GO was approximately $160 \mathrm{~h}$ at the recommended dose of $3 \mathrm{mg} / \mathrm{m}^{2}$. No formal PK studies of GO have been conducted in patients with hepatic or severe renal impairment. Patients with mild-to-moderate renal impairment (creatinine clearance $>30 \mathrm{~mL} / \mathrm{min}$ ) did not show any alterations in pharmacokinetics for GO. [5, $31,32]$

\subsection{Brentuximab Vedotin}

The pharmacokinetics of BV was assessed in phase I trials and in a population analysis of data from 314 patients. $[12,13]$ Typically, $C_{\max }$ was observed closest to the end of intravenous infusion. The observed exposures were dose dependent and the terminal half-life of BV was found to be 4-6 days. There was no accumulation of BV even after repeated doses given every 3 weeks. [12] The average $C_{\max }$ and AUC after a single dose of $1.8 \mathrm{mg} / \mathrm{kg}$ was $31.98 \mu \mathrm{g} / \mathrm{mL}$ and $79.41 \mu \mathrm{g} / \mathrm{mL} \times$ day, respectively. Median $C_{\max }$, AUC, and time to $C_{\max }$ of MMAE, which is a major metabolite of $\mathrm{BV}$, were $4.97 \mathrm{ng} / \mathrm{mL}, 37.03 \mathrm{ng} / \mathrm{mL} \times$ day and 2.09 days, respectively. Subsequent doses of the ADC yielded 50-80\% of the MMAE exposure of the first dose. An equally potent metabolite of MMAE is unlikely to have any impact on systemic effects of MMAE, owing to its substantially lower exposure. The mean steady-state volume of distribution was approximately 6-10 L for the ADC. The typical central and peripheral volume of distribution of MMAE were $7.37 \mathrm{~L}$ and 36.4 L, respectively. MMAE is a CYP3A4 substrate and possibly CYP2D6. However, MMAE inhibits only CYP3A4/5 at levels much higher than were observed during clinical application. Moreover, MMAE did not induce any CYP450 enzymes in vitro. [32]

Brentuximab vedotin is eliminated by catabolism with a typical estimated clearance and half-life of $1.457 \mathrm{~L} /$ day and 4-6 days, respectively. [12] The elimination of MMAE was limited by its rate of release from the ADC. Typical apparent clearance and half-life of MMAE was 19.99 L/day and 3-4 days, respectively. An excretion study was undertaken in patients who received a dose of $1.8 \mathrm{mg} / \mathrm{kg}$ of BV. [12] Approximately $24 \%$ of the total MMAE administered as part of the ADC was recovered in both urine and feces over a 1 -week period. Approximately $72 \%$ was recovered in the feces. The rest (28\%) was excreted in the urine. Baseline serum albumin level was a significant covariate of MMAE clearance. The analysis indicated that MMAE clearance was twofold lower in patients with low serum albumin levels $<3.0 \mathrm{~g} / \mathrm{dL}$ compared with patients with serum albumin levels within the normal range. The latter finding applies to patients with hepatic impairment, evaluated using the Child-Pugh scale. Severe renal impairment with creatinine clearance $<30 \mathrm{~mL} / \mathrm{min}$ resulted in an increase of MMAE exposure by approximately two-fold. [33] In a pediatric population, exposures were approximately dose related, with a trend towards lower ADC exposures at lower ages/ body weights. Median AUC in children and adolescents was approximately $14 \%$ and $3 \%$ lower than in adult patients, respectively, while MMAE exposures were 53\% lower and 
$13 \%$ higher, respectively [34]. Patients who experienced IRRs tested more often positively for ADAs. Nonetheless, the presence of ADAs did not correlate with a significant reduction in serum $\mathrm{BV}$ concentration and did not lead to a decrease in the clinical efficacy of BV.

\subsection{Trastuzumab Emtansine}

Patients who received $3.6 \mathrm{mg} / \mathrm{kg}$ of T-DM1 intravenously every 3 weeks had a mean $C_{\max }$ of $83.4 \mu \mathrm{g} / \mathrm{mL}$. $[18,19]$ The central volume of distribution of T-DM1 was 3.13 L. T-DM1 undergoes deconjugation and catabolism by means of proteolysis in cellular lysosomes. In-vitro studies suggested that the trastuzumab emtansine payload, DM1, is metabolized mainly by CYP3A4 and CYP3A5 and is a P-gp substrate [18]. T-DM1 catabolites MCC-DM1, Lys-MCC-DM1, and DM1 were detected at low levels in human plasma [19]. The clearance of T-DM1 was $0.68 \mathrm{~L} /$ day and its half-life was approximately 4 days. No accumulation of the ADC was observed after repeated dosing every 3 weeks. T-DM1 catabolites were mainly excreted in the bile with minimal elimination in the urine. Mild-to-moderate renal impairment did not alter PK characteristics of T-DM1. No recommendations could be given for patients with creatinine clearance $<30 \mathrm{~mL} / \mathrm{min}$. [18] Plasma concentrations of DM1 and its catabolites were low and comparable between patients with and without hepatic impairment. The AUCs of T-DM1 at cycle 1 in patients with mild or moderate hepatic impairment were approximately $38 \%$ and $67 \%$ lower than that of patients with normal hepatic function, respectively. Patients with severe liver impairment were not evaluated. [19]

\subsection{Inotuzumab Ozogamicin}

In patients with relapsed or refractory acute lymphoblastic leukemia treated with IO, the recommended starting dose of $1.8 \mathrm{mg} / \mathrm{m}^{2} /$ cycle, steady-state exposure was achieved by cycle 4 . $[25,26]$ The mean $C_{\max }$ was $308 \mathrm{ng} / \mathrm{mL}$. The mean total AUC per cycle at steady state was $100 \mu \mathrm{g} / \mathrm{h} /$ $\mathrm{mL}$. The total volume of distribution of IO was $12 \mathrm{~L}$. The calicheamicin derivative is a substrate of P-gp. It is metabolized via non-enzymatic reduction and its concentration in human plasma was found to be below the assay sensitivity. The clearance of IO at steady state was $0.0333 \mathrm{~L} / \mathrm{h}$, and the terminal elimination half-life at the end of cycle 4 was approximately 12.3 days. Between cycles 1 and 4, 5.3-fold accumulation of IO was observed. Based on a population-PK analysis, the clearance of IO in patients with hepatic impairment did not appear to be reduced. Renal function did not alter IO clearance up to a creatinine clearance of $15 \mathrm{~mL} / \mathrm{min}$. There are no data below that threshold.

Increases in QTcF of $\geq 60 \mathrm{~ms}$ from baseline were measured in $3 \%$ patients in the IO arm and $2 \%$ in the control arm (conventional chemotherapy). Increases in $\mathrm{QTcF}$ of $>500 \mathrm{~ms}$ were observed in none of the patients in the IO arm. Mean (90\% confidence interval) maximum QTcF changes from baseline were $16.5 \mathrm{~ms}(14.3-18.7)$ in the IO arm and $10.8 \mathrm{~ms}(8.0-13.6)$ in the investigator's choice of chemotherapy arm. [25] Based on a PK exposure-response analysis in 250 patients who received $1.8 \mathrm{mg} / \mathrm{m}^{2} /$ cycle IO administered as three divided doses on days $1\left(0.8 \mathrm{mg} / \mathrm{m}^{2}\right), 8$ $\left(0.5 \mathrm{mg} / \mathrm{m}^{2}\right)$, and $15\left(0.5 \mathrm{mg} / \mathrm{m}^{2}\right)$ of a 21 - to 28 -day cycle or $1.8 \mathrm{mg} / \mathrm{m}^{2} /$ cycle administered once every 4 weeks, respectively, the median QTcF increased by $2.53 \mathrm{~ms}$ from baseline at the average $C_{\max }$ of $371 \mathrm{ng} / \mathrm{mL}$ and by $3.87 \mathrm{~ms}$ from baseline at a 1.5 times higher average $C_{\max }$ of $569 \mathrm{ng} / \mathrm{mL}$.

\section{Adverse Events}

Common AEs include IRR, from mild allergic reactions to anaphylaxis. Discontinuation of treatment should be considered for patients who develop signs or symptoms of anaphylaxis, including severe respiratory symptoms or clinically significant hypotension. Premedication with a corticosteroid, antihistamine, and acetaminophen is recommended about 1 $\mathrm{h}$ prior to ADC infusion.

\subsection{Gemtuzumab Ozogamicin}

In monotherapy studies, the most common AEs included pyrexia, nausea, infection, chills, hemorrhage, vomiting, thrombocytopenia, fatigue, headache, stomatitis, diarrhea, abdominal pain, and neutropenia, and occurred in more than $30 \%$ of cases. [6] However, clinically relevant serious AEs included neutropenia (34.3\%), thrombocytopenia (21.7\%), and IRRs (2.5\%). The most common AEs (>30\%) in the combination therapy study were hemorrhage and infection. [7] The most relevant AEs in the combination therapy study were severe infection (41.2\%), hemorrhage (9.9\%), hepatotoxicity, including VOD/SOS (3.8\%) and tumor lysis syndrome (1.5\%). Permanent discontinuation of therapy was most often caused by thrombocytopenia, VOD, hemorrhage, and infection in the combination therapy study. Infection, hemorrhage, multi-organ failure, and VOD most frequently led to discontinuation in monotherapy studies. [6] Increased risk for developing VOD was seen in patients who received GO as monotherapy, either before or after HSCT, as well as in patients with moderate-to-severe liver impairment.

In the combination therapy study, VOD was reported in six $(4.6 \%)$ patients during or following treatment, two of these reactions were fatal. The median time from the last GO dose to the onset of VOD was 9 days (range 2-298 days). Patients who received GO as monotherapy and patients who had received an HSCT prior to GO exposure were 2.6 times more likely to develop VOD, compared with patients without 
HSCT prior to treatment with GO. [6] Patients who received an HSCT following treatment with GO were 2.9 times more likely to develop VOD compared with patients without HSCT following treatment with GO. Finally, patients who had moderate or severe liver impairment before treatment were 8.7 times more likely to develop VOD, compared with patients with normal liver function. The patients should be closely monitored for clinical and laboratory signs of liver failure. The ALFA-0701 study recommended a 2-month interval between the administration of GO and HSCT. [10]

Myelosuppression was a very common finding in a study with GO in combination therapy in patients untreated with de novo AML. [7] Grade 3-4 leukopenia, neutropenia, and thrombocytopenia were observed in $100 \%, 96.1 \%$, and $100 \%$, respectively. The median platelet recovery times to counts of $50,000 / \mathrm{mm}^{3}$ and $100,000 / \mathrm{mm}^{3}$ were 34 and 35 days, respectively, during the induction phase, and $83.2 \%$ and $75.6 \%$ of patients achieved platelet recovery counts of $50,000 / \mathrm{mm}^{3}$ and $100,000 / \mathrm{mm}^{3}$, respectively. During the second consolidation course, $97.6 \%$ and $85.4 \%$ of patients had a platelet recovery counts of $50,000 / \mathrm{mm}^{3}$ and $100,000 / \mathrm{mm}^{3}$, respectively. The median platelet recovery times to counts of $50,000 / \mathrm{mm}^{3}$ and $100,000 / \mathrm{mm}^{3}$ were 36.5 and 43 days, respectively. Persistent thrombocytopenia beyond 45 days after the start of therapy occurred in $20.4 \%$ of responding patients. Neutrophil recovery counts to $500 / \mathrm{mm}^{3}$ and $1000 /$ $\mathrm{mm}^{3}$ were documented in $92.4 \%$ and $90.1 \%$ of patients during the induction treatment, respectively, and the median time to achieve it was 25 days.

During the second consolidation course, $97.6 \%$ and $96.3 \%$ of patients had a neutrophil recovery counts of 500/ $\mathrm{mm}^{3}$ and $1000 / \mathrm{mm}^{3}$, respectively. The median neutrophil recovery times to ANC of $500 / \mathrm{mm}^{3}$ and $1000 / \mathrm{mm}^{3}$ were 22 and 27 days, respectively. Severe infections were reported in $77.9 \%$ of patients in the combination therapy study. However, fatal infection was observed in $1.5 \%$ of patients treated with GO and chemotherapy and $2.92 \%$ of patients in the control arm. Grade 3-4 bleeding was observed in $20.6 \%$ of patients. The most frequent hemorrhagic AEs included epistaxis, hemoptysis, hematuria, gastrointestinal bleeding, and pulmonary alveolar hemorrhage. Fatal bleedings were reported in $2.3 \%$ of patients. There have been cases of fatal tumor lysis syndrome complicated by acute renal failure.

Leukoreduction should therefore be considered to reduce leukocyte counts to below $30,000 / \mathrm{mm}^{3}$ prior to $\mathrm{GO}$ administration. Women of childbearing potential and partners of women with childbearing potential should use two methods of contraception during treatment with GO for at least 7 months (female individuals) or 4 months (male individuals) after the last dose. [5] Immunogenicity is a potential complication with all protein-based therapeutics. The occurrence of antidrug antibodies (ADAs) after GO was below $1 \%$ in four clinical studies. [7] However, the impact of ADAs on clinical efficacy and safety cannot be evaluated owing to the low numbers of patients with detectable ADAs. The optimal dose of GO for pediatric patients was not established. In a phase of dose intensification study AAML0531, more patients receiving GO experienced prolonged neutrophil recovery time ( $>59$ days) as compared with the comparator arm ( $12.0 \%$ vs. $6.3 \%)$, and more patients died during remission $(4.2 \%$ vs. $2.4 \%)$. [35] The safety profile was similar to the observed in other studies with GO in adult patients.

\subsection{Brentuximab Vedotin}

The most frequent AEs ( $\geq 10 \%)$ based on the pooled data from studies with BV as monotherapy were infections, peripheral sensory neuropathy, nausea, fatigue, diarrhea, pyrexia, upper respiratory tract infection, neutropenia, rash, cough, vomiting, arthralgia, peripheral motor neuropathy, IRRs, pruritus, constipation, dyspnea, weight decrease, myalgia, and abdominal pain. [36] Peripheral motor neuropathy was observed more often in patients retreated with $\mathrm{BV}$, as compared with phase II studies ( $28 \%$ vs. $9 \%$ ), but was primarily grade 2 . Serious AEs were found in $12 \%$ of patients. The frequency of unique serious adverse drug reactions (ADRs) was $\leq 1 \%$. Twenty-four percent of patients had to stop treatment because of AEs. The safety data in patients retreated with $\mathrm{BV}$ and those treated with $\mathrm{BV}$ who had not received an ASCT were consistent with the safety profile of the pivotal clinical studies. [37]

Grade 3 and 4 neutropenia was reported in 13\% and 5\% of patients, respectively. The median duration of grade 3 or grade 4 neutropenia was limited, with only $2 \%$ of patients presenting with grade 4 neutropenia that lasted $\geq 7$ days. Serious infections and opportunistic infections were reported in $10 \%$ of patients, with sepsis and septic shock in less than $1 \%$ of patients. The most common opportunistic infections included Herpes zoster and Herpes simplex. Treatmentrelated neuropathy was observed in $59 \%$ of patients, with peripheral motor neuropathy affecting $14 \%$ of patients. [12, 13] The median time of onset of this AE was 12 weeks. Patients remained on the treatment for a median of 12 cycles. Treatment discontinuation was reported in $15 \%$ of patients, dose reductions in $15 \%$, and dose delays in $17 \%$ of patients owing to PN. Most patients improved or recovered from this $\mathrm{AE}$ with the median time from onset to resolution from 16 to 23.4 weeks. Patients retreated with BV also presented improvement or resolution of PN symptoms. [38] Infusionrelated reactions including headache, rash, back pain, vomiting, chills, nausea, dyspnea, pruritus, and cough were observed in $13 \%$ of patients. [52]

Anaphylactic reactions have been reported. Premedication with paracetamol, an antihistamine, and a corticosteroid is advised. However, there have been cases of anaphylactic reactions successfully treated with rapid 
desensitization protocols. [39] Such an approach is especially necessary in patients who benefit from the BV treatment. Patients with ADAs were found to have a higher incidence of IRRs. However, the presence of ADA did not result in a decrease in the efficacy of BV. In a limited number of pediatric patients, no new AEs were reported. [40] There have been reports of John Cunningham (JCV) virus-induced progressive multifocal leukoencephalopathy, a frequently fatal John Cunningham virus-induced central nervous system infection. Carson et al. observed five patients with lymphoid malignancies, who developed JCV-induced progressive multifocal leukoencephalopathy after a median of $3 \mathrm{BV}$ doses (range two to six doses) and within a median of 7 weeks (range 3-34 weeks). [41] Two patients with cutaneous T-cell lymphoma had not previously received chemotherapy. Clinical findings included aphasia, dysarthria, confusion, hemiparesis, and gait dysfunction. John Cunningham virus was found in the cerebrospinal fluid (two patients) or central nervous system biopsy (three patients), and brain magnetic resonance imaging revealed white matter abnormalities in all five patients. Four patients died. The sole survivor developed immune reconstitution inflammatory syndrome.

Abdominal pain has been reported in up to $18 \%$ of patients treated with BV. [37] However, pancreatitis had been a previously unidentified serious AE until a fatal case of pancreatitis in a patient receiving BV in a clinical trial was reported. Gandhi et al. reported an additional fatal case and six non-fatal cases of pancreatitis associated with singleagent BV therapy. [42] Cases were collected from lymphoma programs, including AEs reported to the US FDA Adverse Event Report System from June 2011 to July 2013. None of the patients had previous exposure to alcohol, had biliary pathology diagnosed during their ensuing hospital course, or had a history of hypertriglyceridemia. The median time to presentation was 26 days from the first BV dose and 12 days from the most recent treatment. All cases occurred by the third cycle of therapy.

Some pulmonary toxicity has been reported with BV. [37] Cases of pneumonitis, interstitial lung disease, and acute respiratory distress syndrome, including fatal outcomes, have been observed. The phase I study of BV with ABVD or AVD revealed its contraindication with bleomycin due to pulmonary toxicity. [17] A causal relationship with BV could not be established, but the risk cannot be ruled out. The most common AEs reported in the AETHERA trial were PN (67\%), infections (60\%), and neutropenia (35\%). [43] Most PN cases (57\%) required dose delays and reductions. The median time to PN onset was 13.7 weeks (range 1-47.4 weeks). Peripheral neuropathy continued to resolve after the end of treatment, reaching similar symptom resolution as in the placebo arm at 3 years of follow-up. Brentuximab vedotin had no significant impact on the pre-existing
PN. Neutropenia was managed with dose delays and/or granulocyte-colony stimulating factor, without dose reductions or discontinuations.

Some other less prevalent but serious AEs, including pulmonitis, hepatotoxicity, and cardiotoxicity, were rare in both arms and were managed with BV dose modifications or discontinuations. Very few patients with comorbidities or risk factors developed secondary malignancies. Alig et al. described a case of severe cytokine release syndrome resulting in fever, chills, dyspnea, and catecholamine-dependent shock occurring immediately after the first dose of BV in a 64-year-old male Caucasian patient with early relapsed, anaplastic lymphoma kinase-negative systemic anaplastic large cell lymphoma, who had undergone autologous stem cell transplantation. [44] Restaging revealed a mediastinal and retroperitoneal lymphadenopathy as well as a pulmonary manifestation of the lymphoma. As premedication, the patient received intravenous dimetindene. The application of prednisolone was omitted to prevent corticosteroid-mediated CD30 downregulation. Brentuximab vedotin was infused over $30 \mathrm{~min}$ at the dose of $1.8 \mathrm{mg} / \mathrm{kg}$. Approximately $60 \mathrm{~min}$ after the start of the infusion, the patient developed severe chills, fever, and moderate dyspnea. The patient also became oliguric and hypotensive. Laboratory results revealed lactate acidosis, a sudden increase in lactate dehydrogenase and interleukin-6 levels as well as a decline in leukocyte count, with a normal level of uric acid. The patient recovered approximately $18 \mathrm{~h}$ after BV infusion. Three weeks later, BV therapy was continued after premedication (prednisolone, dimetindene, ranitidine, acetaminophen) with no further complications.

The abovementioned case report demonstrates a risk for cytokine release syndrome in patients with high tumor burden, even though the main mode of BV action is not complement or cell mediated. However, the higher number of CD30-positive cells in anaplastic large cell lymphoma may contribute to the development of cytokine release syndrome.

\subsection{Trastuzumab Emtansine}

The most common serious ADRs evaluated in a group of 1871 patients were hemorrhage, pyrexia, dyspnea, musculoskeletal pain, thrombocytopenia, abdominal pain, and vomiting. $[18,45]$ The most common ADRs ( $\geq 25 \%)$ with T-DM1 were nausea, fatigue, and headache. The majority of them were grade 1 or 2 . The most common National Cancer Institute - Common Terminology Criteria for Adverse Events grade $\geq 3$ ADRs $(>2 \%$ ) were thrombocytopenia, increased transaminases, anemia, neutropenia, fatigue, hypokalemia, musculoskeletal pain, and hemorrhage. There have been reports on serious hepatobiliary disorders, with at least two fatal cases of liver failure with encephalopathy in clinical trials. [24, 45, 46] Liver toxicity is usually 
asymptomatic and presents as increased serum transaminases. T-DM1 should be permanently discontinued if serum transaminases increase more than three times and total bilirubin is increased more than two times the upper normal limit. Nodular regenerative hyperplasia of the liver has been observed in three cases out of 884 patients. The injury may develop within months of starting the treatment, especially in patients with previous multiple chemotherapy regimens. Nodular regenerative hyperplasia may lead to non-cirrhotic portal hypertension. It is the maytansinoid derivative responsible for this type of toxicity. [47] Upon diagnosis of nodular regenerative hyperplasia, T-DM1 treatment must be permanently discontinued.

Another rare complication that might need careful approach is a decrease of left ventricular ejection fraction (LVEF) to $<40 \%$. [18] This AE is specifically related to trastuzumab. A retrospective study of 246 patients treated with trastuzumab revealed a LVEF decline $\geq 10 \%$ in $13 \%$ of patients, $\geq 20 \%$ decline in $4.5 \%$ of cases within 1 year after trastuzumab initiation. [48] Trastuzumab was discontinued because of suspected cardiotoxicity in $2.4 \%$ of patients. Moreover, almost $20 \%$ of patients experienced symptoms related to cardiotoxicity during therapy, and that accumulated among those with LVEF drop. A decrease in LVEF, as well as trastuzumab discontinuation, were related to the underlying cardiovascular diseases and multiple cardiac medications. The majority of LVEF worsening and trastuzumab discontinuations were observed within 6 months of treatment initiation. Most patients recovered their LVEF drop to $<10 \%$ of the baseline and their survival was not hampered. Left ventricular dysfunction has been observed in $1.8 \%$ of patients receiving T-DM1, compared with $3.3 \%$ of patients treated with lapatinib and capecytabine. [48] Any decrease of LVEF to below $40 \%$ or to $40-45 \%$ with at least a $10 \%$ decrease mandates withholding of T-DM1 or even stopping it in case there is no improvement within 3 weeks.

T-DM1 can pose harm to a fetus when administered to a pregnant woman or within 7 months prior to conception. [49] There have been reports on several complications including oligohydramnios with its sequelae, and fetal/neoatal death with trastuzumab. Forty-two percent of abnormally low amniotic fluid during chemotherapy in pregnant women was related to gestational exposure to trastuzumab in the second or third trimester. The cytotoxic component of T-DM1, DM1, can cause embryo-fetal toxicity based on its mechanism of action.

Cases of interstitial lung disease have been described in clinical trials. [45] However, the incidence of this complication is low at $0.8 \%$ (seven out of 884 treated patients). Perhaps it is due to infusion reactions but such analysis has not been conducted because of the low number of cases. The overall incidence of IRRs was $1.4 \%$. In most cases, these reactions resolved within hours or up to a day after stopping the infusion.

Another potential complication are hemorrhagic events, which occurred in up to $32.2 \%$ of patients treated with T-DM1, compared with $16.4 \%$ of patients receiving lapatinib and capecitabine. [46] The incidence of grade 3 and up hemorrhage was $1.8 \%$ in the T-DM1 arm and $0.8 \%$ in the control arm. Anticoagulation therapy, antiplatelet therapy, or thrombocytopenia could contribute to some bleeding episodes but not all. Therefore, caution is advised when concomitant agents are used. Thrombocytopenia after T-DM1 was found in up to $31.2 \%$ of patients, compared with $3.3 \%$ in the lapatinib plus capecitabine group. [46] Grade $\geq 3$ low platelet count was reported in $14.5 \%$ of patients receiving T-DM1, and $0.4 \%$ receiving lapatinib plus capecitabine. Nadir of platelet counts was usually observed by day 8 and would resolve before the next scheduled dose. Peripheral neuropathy was reported in $21.1 \%$ of patients taking T-DM1 and $13.5 \%$ in the lapatinib plus capecitabine-treated group. [46] The incidence of grade $\geq 3$ PN was $2.2 \%$ in the T-DM1treated group and $0.2 \%$ in the lapatinib plus capecitabinetreated group. T-DM1 has the potential to elicit immune responses. Following T-DM1 dosing, 5.3\% of patients had anti-T-DM1 antibodies in their serum. [45] However, the neutralizing properties of these antibodies have not been studied and their clinical significance is unknown.

\subsection{Inotuzumab Ozogamicin}

The most common $(\geq 20 \%)$ AEs were thrombocytopenia $(51 \%)$, neutropenia (49\%), infection (48\%), anemia (36\%), leukopenia (35\%), fatigue (35\%), hemorrhage (33\%), pyrexia (32\%), nausea (31\%), headache (28\%), febrile neutropenia (26\%), increased transaminases (26\%), abdominal pain (23\%), increased gamma-glutamyltransferase (21\%), and hyperbilirubinemia $(21 \%)$. [26, 27] The most common (2\%) serious AEs were infection (23\%), febrile neutropenia (11\%), hemorrhage (5\%), abdominal pain (3\%), pyrexia (3\%), VOD/SOS (2\%), and fatigue (2\%). Veno-occlusive disease/sinusoidal obstruction syndrome was observed in $13 \%$ of patients out of 164, including five who did not proceed to HSCT. Veno-occlusive disease/sinusoidal obstruction syndrome was reported in another $22 \%$ of patients out of 77 , who underwent HSCT. [26, 27] Five cases after HSCT were fatal. This AE was reported up to 56 days after the last dose of IO as the sole therapy, and the median time after HSCT preceded by $\mathrm{InO}$ was 15 days. Other risk factors included a conditioning regimen containing two alkylating drugs, age $\geq 5$ years, and serum bilirubin $\geq$ upper limit of normal prior to HSCT.

In the INO-VATE ALL trial, a higher post-HSCT nonrelapse mortality rate was observed in patients receiving IO compared with the investigator's choice of chemotherapy 
arm, which resulted in a higher day 100 post-HSCT mortality rate. [27] Overall, $48 \%$ of patients in the IO arm and $22 \%$ of patients in the investigator's choice of chemotherapy arm had a follow-up HSCT. The post-HSCT non-relapse mortality rate was 39\% and $23 \%$ in the InO arm compared with the investigator's choice of chemotherapy arm, respectively. Finally, in the IO arm, the most common causes of post-HSCT nonrelapse mortality included VOD and infections. Hyperbilirubinemia and increased transaminases were reported in $21 \%$ and $26 \%$ of patients, respectively. [49] The median time to onset of hyperbilirubinemia and increased transaminases was 73 days and 29 days, respectively. Six and seven percent of patients presented grade $\geq 3$ hyperbilirubinemia and increased transaminases, respectively.

In a safety analysis in 164 patients, thrombocytopenia and neutropenia were reported in $51 \%$ and $49 \%$ of patients, respectively. [50] Grade 3 thrombocytopenia and neutropenia were observed in $14 \%$ and $20 \%$ of patients, respectively. Grade 4 thrombocytopenia and neutropenia were reported in $28 \%$ and $27 \%$ of patients, respectively. Febrile neutropenia, which may be life threatening, was reported in $43(26 \%)$ patients. [50] The overall incidence of infections, including serious infections with fatal outcome in the pivotal study with IO was $48 \%$ of patients. Fatal infections, including pneumonia, neutropenic sepsis, sepsis, septic shock, and pseudomonal sepsis, were reported in 5\% of patients. Thirty-three percent of patients experienced some, mostly mild bleeding including epistaxis, upper gastrointestinal hemorrhage, lower gastrointestinal hemorrhage, and central nervous system hemorrhage. [50] Grade 3 and 4 hemorrhagic events were reported in 5\% of patients. Infusion-related reactions were observed in $10 \%$ of patients and were all of less than grade 2 in severity. Infusionrelated reactions typically occurred in cycle 1 and resolved with no further complications. [50] Tumor lysis syndrome was reported in $2 \%$ of patients. It occurred shortly after the end of IO infusion and resolved with standard treatment [50]. No grade $\geq 3$ QT interval prolongation was observed in the pivotal study with 164 participants. [50] Increases in QT inter$\mathrm{val} \geq 60 \mathrm{~ms}$ from baseline were reported in $3 \%$ of patients. No patient had QT interval corrected for heart rate using Fridericia formula values that exceeded $500 \mathrm{~ms}$. There were cases of increased amylase and lipase in 5\% and 9\% of patients form the pivotal study. [50] Grade $\geq 3$ amylase and lipase increases were reported in $2 \%$ and $4 \%$ of patients, respectively. Three percent of patients were reported to have anti-IO antibodies. There were no neutralizing anti-IO antibodies detected and no effect on IO clearance was found. [50] The effect of these antibodies on clinical activity and safety of IO could not be assessed because of small numbers of patients.

\section{Drug Interactions}

Antibody-drug conjugates carry active cytotoxic components that undergo metabolic changes as any other medicinal product. Therefore, there is potential for drug-drug interactions.

\subsection{Gemtuzumab Ozogamicin}

Gemtuzumab ozogamicin and its payload, $N$-acetyl gamma calicheamicin dimethyl hydrazide, had little inhibitory effect on CYP1A2, CYP2A6 (tested only using GO), CYP2B6, CYP2C8, CYP2C9, CYP2C19, CYP2D6, and CYP3A4/5 at clinically relevant concentrations in vitro. $[5,51] \mathrm{N}$-acetyl gamma calicheamicin dimethyl hydrazide and GO had also a low potential to induce the activities of CYP1A2, CYP2B6, and CYP3A4 at clinically relevant concentrations. In vitro, $\mathrm{N}$-acetyl gamma calicheamicin dimethyl hydrazide had a low potential to inhibit the activities of UGT enzymes at clinically relevant concentrations. $N$-acetyl gamma calicheamicin dimethyl hydrazide had a low potential to inhibit the activities of P-gp, breast cancer resistance protein, bile salt export pump, multidrug resistance associated protein 2 , multidrug and toxin extrusion protein 1 and multidrug and toxin extrusion protein 2, organic anion transporter 1 and organic anion transporter, organic cation transporter 1 and organic cation transporter 2 , and organic anion transporting polypeptide (OATP) 1B1 and organic anion transporter 1B3 at clinically relevant levels in vitro. Co-administration of GO with daunorubicine and cytarabine was not predicted to alter the pharmacokinetics of any of these agents. [5]

\subsection{Brentuximab Vedotin}

Brentuximab vedotin is a substrate of CYP3A4 and possibly CYP2D6. [52] The mAb portion of the drug is catabolized as a protein. The payload, MMAE, is metabolized via oxidation by CYP3A4/5. It does not inhibit other isoforms. Strong CYP3A4 and P-gp inhibitors (e.g., ketoconazole, itraconazole, clarithromycin, atazanavir, indinavir, nefazodone, nelfinavir, ritonavir, saquinavir, telithromycin, and voriconazole) were found to increase MMAE exposure by $73 \%$ but did not change the plasma exposure to BV. [33] Co-administration of BV with strong inhibitors may lead to increased incidence of neutropenia. Rifampicin, a strong CYP3A4 inducer, did not alter the plasma exposure to BV but reduced plasma concentrations of MMAE metabolites. Midazolam, a CYP3A4 substrate, did not alter its metabolism when administered concomitantly with BV. [33, 53] 


\subsection{Trastuzumab Emtansine}

In-vitro studies suggest that DM1, the cytotoxic part of T-DM1, is metabolized mainly by CYP3A4 and, to a lesser extent, by CYP3A5. [18] Strong CYP3A4 inhibitors (e.g., ketoconazole, itraconazole, clarithromycin, atazanavir, indinavir, nefazodone, nelfinavir, ritonavir, saquinavir, telithromycin, and voriconazole) should be avoided with Kadcyla, as there is a potential for an increase in DM1 exposure and toxicity. [54, 55]

\subsection{Inotuzumab Ozogamicin}

Co-administration of IO with inhibitors or inducers of cytochrome P450 or UGT drug-metabolizing enzymes were found unlikely to alter the exposure to $\mathrm{N}$-acetylgamma-calicheamicin dimethylhydrazide, based on invitro data. [25, 51] Moreover, IO and its calicheamicin derivative are unlikely to change the exposure of CYP enzyme substrates and substrates of UGT enzymes or major drug transporters. The concomitant use with medicines known to prolong QT interval or to induce torsades de pointes arrhythmia should be thoroughly considered and the patient requires QT interval monitoring. [25]

\section{New Agents under Development: Indications Pursued and Current Status}

Antibody-drug conjugates pose a promising therapeutic option for patients with malignancies. Therefore, new molecules are being thoroughly studied in many human neoplastic diseases. Below is an example of a few of them (Table 2).

\subsection{Glembatumumab Vedotin}

Glembatumumab vedotin (GV, CDX-011; Celldex, USA) is a fully human monoclonal ADC targeting glycoprotein NMB (gpNMB). This protein is overexpressed by multiple tumor types, including breast cancer, melanoma, lung cancer, uveal melanoma, and osteosarcoma. The antibody is conjugated to MMAE via a stable linker. [56]

In a randomized phase II EMERGE study in advanced gpNMB-expressing breast cancer, GV was well tolerated compared with the investigator's choice chemotherapy. [57] Eighty-three patients received GV $1.88 \mathrm{mg} / \mathrm{kg}$ intravenously every 3 weeks. The investigator's choice treatment was administered to 41 patients and included one of the following: eribulin, capecitabine, vinorelbine, gemcitabine, docetaxel, paclitaxel, albumin-bound paclitaxel, doxorubicin, liposomal doxorubicin, and ixabepilone. The median number of cycles was two cycles in both arms, with a range of 1-15 cycles of GV and 1-14 of investigator's choice treatment. Glembatumumab vedotin was well tolerated as compared with the investigator's choice chemotherapy. Unplanned analysis demonstrated ORR of $18 \%$ vs. $0 \%$ in patients with triple-negative breast cancer, and $40 \%$ vs. $0 \%$ in gpNMBoverexpressing triple-negative breast cancer. The conclusion from the study was that although the primary endpoint in advanced gpNMB-expressing breast cancer was not met for all enrolled patients, activity of GV may be enhanced in patients with gpNMB-overexpressing tumors and/or triplenegative breast cancer.

A pivotal randomized phase II METRIC study compared $\mathrm{GV}$ with capecitabine in patients with metastatic triplenegative breast cancers that overexpress gpNMB. [58] In total, 327 patients were enrolled into METRIC. The primary endpoint of the study was PFS. The secondary endpoints of response rate, OS, duration of response, and safety were also important in assessing clinical benefit. This trial failed to meet its primary endpoint, PFS (hazard ratio $=0.95$; median PFS: GV 2.9 months vs. capecitabine 2.8 months; $p=0.76$ ). Moreover, researchers did not observe a significant advantage for GV in the key secondary endpoints, including ORR, duration of response, and OS. Given the results, GV is not in the pipeline of Celldex anymore, although some clinical trials are still ongoing (Table 2).

\subsection{Gsk2857916}

GSK2857916 (GlaxoSmithKline, UK) comprises a humanized anti-B-cell maturation antigen (BCMA) mAb conjugated to the cytotoxic agent monomethyl auristatin $\mathrm{F}$ via a non-cleavable linker. [58] B-cell maturation antigens are cell surface receptors in the tumor necrosis factor superfamily, which expression is restricted to B lineage cells at later stages of differentiation, and is required for the survival of long-lived plasma cells. Its function is to promote plasma cell survival by transduction of signals from two known ligands, BAFF and APRIL. However, its activity is also important for myeloma cell growth and survival. The BCMA is expressed at varying levels in patients with myeloma. [59] GSK2857916 was studied in patients with relapsed/ refractory multiple myeloma and other advanced hematologic malignancies expressing BCMA in the open-label dose-escalation study DREAMM-1 to investigate its safety, pharmacokinetics, pharmacodynamics, immunogenicity, and clinical activity. [60, 61] Patients were enrolled in this study independent of BCMA expression levels.

In part 1 of the dose-escalation study, the participants $(n=38)$ were heavily pre-treated. [60] Eligible subjects must have had prior treatments with alkylators, proteasome inhibitors, immunomodulators, and stem cell transplantation, if 
Table 2 New antibody-drug conjugates (ADCs) in clinical trials

New ADC Target molecule Clinical trials (ClinicalTrials.gov identifier)

Glembatumumab vedotin Anti-NMB glycoprotein 1. Study of glembatumumab vedotin in gpNMB-expressing, advanced, or metastatic SCC of the lung (NCT02713828)

2. Glembatumumab vedotin in treating patients with metastatic or locally recurrent uveal melanoma (NCT02363283)

3. A study of glembatumumab vedotin as monotherapy or in combination with immunotherapies in patients with advanced melanoma (NCT02302339)

4. Glembatumumab vedotin in treating patients with recurrent or refractory osteosarcoma (NCT02487979)

GSK2857916 Anti-BCMA

Sacituzumab govitecan Anti-TROP-2

Anetumab ravtansine Anti-mesothelin

Trastuzumab deruxtecan Anti-HER2

Coltuximab ravtansine Anti-CD19
1. Dose escalation study to investigate the safety, pharmacokinetics, pharmacodynamics, immunogenicity and clinical activity of GSK2857916 (NCT02064387)

2. A DREAMM-2 study to investigate the efficacy and safety of two doses of GSK2857916 in subjects with multiple myeloma who have failed prior treatment with an anti-cd38 antibody (NCT03525678)

3. To evaluate safety, tolerability, and clinical activity of the antibody-drug conjugate, GSK2857916 administered in combination with lenalidomide plus dexamethasone (arm A), or in combination with bortezomib plus dexamethasone (arm B) in subjects with relapsed/refractory multiple myeloma (RRMM) (NCT03544281)

1. ASCENT - study of sacituzumab govitecan in refractory/relapsed triple-negative breast cancer (NCT02574455)

2. Phase $1 / 2$ study of IMMU-132 in patients with epithelial cancers (NCT01631552)

3. Phase 2 open label, study of IMMU-132 in metastatic urothelial cancer (nCT03547973)

1. Phase $1 \mathrm{~b}$ multi-indication study of anetumab ravtansine in mesothelin expressing advanced solid tumors (NCT03102320)

2. Phase $1 \mathrm{~b}$ study of anetumab ravtansine in combination with pegylated liposomal doxorubicin in patients with recurrent mesothelin-expressing platinum-resistant cancer (NCT02751918)

3.Phase 2anetumab ravtansine in pre-treated mesothelin-expressing pancreatic cancer (NCT03023722)

4. Bevacizumab and anetumab ravtansine or paclitaxel in treating participants with refractory ovarian, fallopian tube, or primary peritoneal cancer (NCT03587311)

5 . Phase $1 \mathrm{~b}$ study of anetumab ravtansine in combination with pemetrexed and cisplatin in mesothelin-expressing solid tumors (NCT02639091)

1. DS-8201a versus T-DM1 for human epidermal growth factor receptor 2 (HER2)-positive, unresectable and/or metastatic breast cancer previously treated with trastuzumab and taxane (NCT03529110)

2. Phase 2, multicenter, open-label study of DS-8201a, an anti-HER2-antibody drug conjugate (ADC) for HER2-positive, unresectable and/or metastatic breast cancer subjects previously treated with T-DM1 (NCT03248492)

3. Phase 2, multicenter, open-label study of DS-8201a in subjects with HER2-expressing advanced gastric or gastroesophageal junction adenocarcinoma (NCT03329690)

4. Phase 2, multicenter, open-label study of DS-8201a in subjects with HER2-expressing advanced colorectal cancer (NCT03384940)

5. Phase 2, multicenter, open-label, 2-cohort study of trastuzumab deruxtecan (DS-8201a), an anti-HER2 antibody drug conjugate (ADC), for HER2-over-expressing or -mutated, unresectable and/or metastatic non-small cell lung cancer (NSCLC) (NCT03505710)

6. Phase 1, two-part, multicenter, non-randomized, open-label, multiple dose first-in-human study of DS-8201A, in subjects with advanced solid malignant tumors (NCT02564900)

7. DS-8201a versus investigator's choice for HER2-positive, unresectable and/or metastatic breast cancer pretreated with prior standard of care (NCT03523585)

1. SAR3419 as single agent in relapsed-refractory diffuse large B-cell lymphoma (DLBCL) patients (NCT01472887)

2. SAR3419 in acute lymphoblastic leukemia (NCT01440179)

3. SAR3419 administered weekly in patients with relapsed/refractory CD19-positive B-cell non-Hodgkin's lymphoma (NCT00796731)

4. Multi-dose-escalation safety and pharmacokinetic study of SAR3419 as single agent in relapsed/refractory B-cell non-Hodgkin's lymphoma (NCT00549185)

5. Combination of SAR3419 and rituximab in relapsed/refractory diffuse large B-cell lymphoma (NCT01470456)

$B C M A$ anti-B-cell maturation antigen, $N M B$ 
transplant eligible. Part 2 of the study enrolled 35 patients, who received GSK2857916 at a dose of $3.4 \mathrm{mg} / \mathrm{kg}$ intravenously every 3 weeks. [61] Fifty-seven percent of the patients had at least five prior lines of treatment and $40 \%$ had prior daratumumab treatment. Patients remained on the treatment until progression, unacceptable toxicity, consent withdrawal, or completing 16 treatment cycles. In the doseescalation phase of the study, 23 patients out of 24 (96\%) experienced AEs. The most frequent $(\geq 20 \%)$, regardless of cause, were nausea (42\%), fatigue (38\%), anemia (29\%), chills $(29 \%)$, pyrexia (29\%), thrombocytopenia (29\%), dry eye $(21 \%)$, and hypercalcemia $(21 \%)$. Grade $3 / 4$ AEs reported in $\geq 10 \%$ of patients were thrombocytopenia, anemia, and neutropenia. The study results did not show doselimiting toxicities. The results presented at the American Society of Hematology 2017 annual meeting demonstrate that GSK2857916 monotherapy offers a 60\% ORR and a median PFS of 7.9 months. [61] Currently, there is an ongoing study with GSK2857916 DREAMM-2 with estimated enrollment of 155 participants (Table 2). GSK2857916 was granted PRIME and Breakthrough Therapy designations in 2017 by the EMA and FDA, respectively.

\subsection{Sacituzumab Govitecan}

Sacituzumab govitecan (IMMU-132; Immunomedics, USA) is an anti-TROP- $2 \mathrm{mAb}$ conjugated with an active metabolite of irinotecan (SN-38) via a pH-sensitive linker. [62] TROP-2 is a receptor that can be found on many human cancer cells, such as of the breast, cervix, colon and rectum, kidney, liver, lung, ovary, pancreas, and prostate, but with only limited expression in normal human tissues. Results from an interim phase II study with IMMU-132 showed that the investigational agent was active in patients with metastatic urothelial cancer who have relapsed or are refractory to chemotherapies and immune checkpoint inhibitors. [63] In the singlearm phase II study with sacituzumab govitecan, a total of 41 patients with metastatic urothelial cancer were enrolled to receive $10 \mathrm{mg} / \mathrm{kg}$ of sacituzumab govitecan on days 1 and 8 of 3-week cycles. Grade 3 or higher AEs were limited to neutropenia (39\%), anemia (10\%), diarrhea (7\%), and fatigue (7\%). The confirmed ORR among 41 patients was $34 \%$, including two confirmed CRs and 12 confirmed PRs. IMMU-132 also proved its safety and efficiency in a phase II trial in patients with triple-negative breast cancer. [64] In 69 patients, the ORR was 30\%. Median PFS and OS were 6 and 16.6 months, respectively. Grade $\geq 3$ AEs included neutropenia (39\%), leukopenia (16\%), anemia (14\%), and diarrhea (13\%). Currently, sacituzumab govitecan is being studied in several clinical trials (Table 2).

\subsection{Anetumab Ravtansine}

Anetumab ravtansine (AR, BAY 94-9343; Bayer, Germany) is an ADC consisting of a human anti-mesothelin antibody conjugated to the maytansinoid tubulin inhibitor DM4 via a disulfide-containing linker. [65] Mesothelin is over-expressed on all mesotheliomas as well as many ovarian and pancreatic cancers while minimally expressed on normal tissue. Moreover, mesothelin expression was found in $50 \%$ of lung adenocarcinomas, $60 \%$ of gastric cancers, and $67 \%$ of triple-negative breast cancer. [65] Anetumab ravtansine has demonstrated potent and selective cytotoxicity of mesothelin-expressing cells in vitro, with a $50 \%$ inhibitory concentration (IC50) of $0.72 \mathrm{nmol} / \mathrm{L}$, without affecting mesothelin-negative or non-proliferating cells. Anetumab ravtansine inhibited tumor growth in xenograft models. Furthermore, it was able to induce a bystander effect on surrounding mesothelin-negative tumor cells. [65]

In a phase I clinical trial in patients with advanced solid tumors, AR demonstrated promising efficacy with durable responses in patients with malignant pleural mesothelioma, and a manageable safety profile. [66] The phase I trial results showed that out of the 16 mesothelioma patients treated at the maximum tolerated dose, five (31\%) had objective tumor reduction, while seven (44\%) achieved stable disease. In most patients, the response was very durable with three subjects still receiving the drug more than 2 years after starting the therapy. There were no DLTs observed up to a dose of $5.5 \mathrm{mg} / \mathrm{kg}$. There was only one grade 3 severe AE of chest pain possibly related to the study drug, and there were no other drug-related high-grade AEs. The most common grade 1 and 2 AEs were fatigue, nausea, anorexia, vomiting, and weakness.

Anetumab ravtansine was also investigated in a randomized open-label superiority phase II study (NCT02610140) as second-line treatment in patients with advanced or metastatic mesothelin-positive malignant pleural mesothelioma, whose disease had progressed after treatment with first-line platinum/pemetrexed-based chemotherapy. [67] The trial randomized 248 patients in a 2:1 ratio to receive either AR $(6.5 \mathrm{mg} / \mathrm{kg}$ intravenously every 3 weeks $)$ or vinorelbine $\left(30 \mathrm{mg} / \mathrm{m}^{2}\right.$ intravenously every week). The primary endpoint of this trial was PFS. Secondary endpoints included OS, as well as patient-reported outcomes, objective tumor response rate, duration of response, disease control rate, and durable response rate. However, this phase II clinical trial did not meet its primary endpoint of PFS, as it was published on the official Bayer website. [68] However, the company remain committed to further evaluating the utility and safety of AR across multiple tumor types. Anetumab ravtansine is currently being investigated in a variety of other mesothelin-positive tumors, including a phase I multi-indication study in six different types of advanced 
solid tumors, a phase $\mathrm{Ib}$ combination-study in patients with recurrent platinum-resistent ovarian cancer, as well as a phase II study in patients with mesothelin-expressing pancreatic cancer (Table 2).

\subsection{Trastuzumab Deruxtecan}

Trastuzumab deruxtecan (DS-8201; Daiichi Sankyo, Japan) binds to HER2, triggering an antibody-dependent cell cytotoxic response, then is internalized and broken down in lysosomes to release Dxd topoisomerase I inhibitor. [69] In-vitro and in-vivo pharmacologic activities of DS-8201a were evaluated and compared with T-DM1 in several HER2positive cell lines and patient-derived xenograft models. [70] DS-8201a proved its great potential to respond to T-DM1-insensitive HER2-positive cancers and low HER2expressing cancers. DS-8201 was studied in a phase I trial in patients with advanced/unresectable or metastatic breast cancer, gastric or gastroesophageal junction adenocarcinoma, or other solid tumors that is/are refractory to or intolerable with standard treatment or for which no standard treatment is available.

In part 1 of the study, DS-8201 a was given as an intravenous infusion every 3 weeks at doses ranging from 0.8 to $8.0 \mathrm{mg} / \mathrm{kg}$. [71] Twenty-two patients were enrolled in this study, including 16 patients with breast cancer, five patients with gastric cancer, and one patient with gastroesophageal junction adenocarcinoma. There were 13 subjects with breast cancer previously treated with T-DM1. There were no dose-limiting toxicities, and the MTD was not reached. The most common AEs were grade $<3$ gastrointestinal and hematologic toxicities. Patients with breast cancer refractory to T-DM1 achieved disease control, with five patients demonstrating PR. Seven grade 3 AEs were reported in three patients (one hypokalemia, one anemia, one neutropenia, two lymphopenia, one alkaline phosphatase increase, and one cholangitis). The dose of $6.4 \mathrm{mg} / \mathrm{kg}$ tri-weekly was preliminarily selected as the recommended phase II dose.

The second part of this phase I study is currently evaluating DS-8201 in patients with advanced/unresectable or metastatic solid tumors that are refractory or intolerant to standard treatment, or for whom no standard treatment is available. [72] Updated preliminary subgroup analysis results in 44 of 45 efficacy evaluable patients with HER2expressing gastric cancer or gastroesophageal junction adenocarcinoma previously treated with trastuzumab and chemotherapy showed that DS-8201 demonstrated a confirmed ORR of $45.5 \%$ and a disease control rate of $81.8 \%$. [73] The most common ( $>30 \%)$ AEs of any grade included nausea $(71.1 \%)$, decreased appetite $(64.4 \%)$, thrombocytopenia (33.3\%), leukopenia (33.3\%), and constipation (31.1\%). Grade 3 AEs occurring in $>10 \%$ of patients included anemia (24.4\%), neutropenia (15.6\%), and thrombocytopenia
(13.3\%). Grade 4 AEs included thrombocytopenia, leukopenia, and neutropenia ( $4.4 \%$ each). There were two potential grade 1 and 3 cases of interstitial lung disease. The longterm phase I safety and efficacy data for DS-8201 in 241 heavily pretreated patients with HER2-expressing breast, gastric, and other solid cancers who received recommended expansion doses of $5.4 \mathrm{mg} / \mathrm{kg}$ or $6.4 \mathrm{mg} / \mathrm{kg}$ were presented at the 2018 American Society of Clinical Oncology meeting. [74]

DS-8201 is currently in a pivotal phase II clinical trial for HER2-positive unresectable and/or metastatic breast cancer resistant or refractory to T-DM1 (DESTINY-Breast01), pivotal phase II development for HER2-positive advanced gastric cancer resistant or refractory to trastuzumab (DESTINY-Gastric01), phase II development for HER2-expressing advanced colorectal cancer, phase II development for unresectable and/or metastatic non-squamous HER2-overexpressing or HER2-mutated non-small-cell lung cancer, and phase I development for other HER2-expressing advanced/ unresectable or metastatic solid tumors.

DS-8201 has been granted breakthrough therapy designation for the treatment of patients with HER2-positive, locally advanced or metastatic breast cancer who have been treated with trastuzumab and pertuzumab and have disease progression after T-DM1. DS-8201 has been granted fast track designation for the treatment of HER2-positive unresectable and/or metastatic breast cancer in patients who have progressed after prior treatment with HER2-targeted therapies including T-DM1 by the FDA. DS-8201 has also been granted SAKIGAKE Designation by the Japan Ministry of Health, Labour and Welfare for the treatment of HER2-positive advanced gastric or gastroesophageal junction cancer.

\subsection{Coltuximab Ravtansine}

Coltuximab ravtansine (SAR3419; Sanofi/Immunogen, USA) is a humanized anti-CD19 mAb linked to DM4, a maytansine analog. It has been studied in patients with relapsed or refractory CD19-positive non-HL. Clinical evidence of its activity was assessed in 39 patients with relapsed or refractory CD19-positive non-HL. [75] The patients were administered SAR3419 every 21 days at doses ranging from 10 to $270 \mathrm{mg} / \mathrm{m}^{2}$. The DLTs included reversible but severe blurred vision caused by epithelial corneal lesions in six subjects and sensory PN in one patient. The MTD was $160 \mathrm{mg} / \mathrm{m}^{2}$ given every 3 weeks. Twenty-six patients demonstrated a reduction in the tumor size, with six of them showing PR or CR. Patients refractory to rituximab also benefited from the treatment. The pharmacokinetics of SAR3419 was linear, with low clearance.

The second phase I study was initiated with a lower SAR3419 dose. [76] The planned starting dose level was $10 \mathrm{mg} / \mathrm{m}^{2} /$ week corresponding to a total dose of $30 \mathrm{mg} / \mathrm{m}^{2}$ 
of SAR3419 administered over a 3-week period in the first phase I study. Forty-four patients received doses ranging from 5 to $70 \mathrm{mg} / \mathrm{m}^{2}$ repeatedly weekly for $8-12$ doses. The schedule was optimized, based on the safety profile, to four weekly doses, followed by four biweekly doses of $55 \mathrm{mg} / \mathrm{m}^{2}$. The recommended dose was chosen with the intent to reduce drug accumulation, with SAR3419 presenting nearly a 7-day half-life and a low clearance of $0.6 \mathrm{~L} /$ day. The rationale for this schedule was based on the clinical evidence of grade 3 PNs with late onset (weeks 7 and 8) during the weekly schedule, supported by preliminary PK data showing accumulation of the ADC, with a steady state reached after four weekly administrations. One patient developed grade 3 optic neuropathy and one patient had grade 3 hand and feet paresthesias. [76] Both events recovered within 6 weeks, although the latter lead to treatment discontinuation. The most frequent treatment-related AEs were gastrointestinal disorders in $27 \%$ of patients, eye disorders in $23 \%$ of patients mainly including blurred vision, and fatigue in $23 \%$ of patients. Grade 3 and higher AEs were reported in 32\% of patients, and included cholestasis, sensory PN, elevated liver enzymes, pneumonia, allergic alveolitis, and optic neuropathy. A case of fatal progressive multifocal leukoencephalopathy was reported during the follow-up visits in one patient who received SAR3419 at the dose of $14 \mathrm{mg} /$ $\mathrm{m}^{2}$ weekly for eight doses as fourth-line therapy. However, prior lymphoma treatment involved a 17 -month exposure to rituximab. The overall anti-lymphoma activity was observed with both schedules in approximately $30 \%$ of patients with either indolent or aggressive disease. [75, 76]

The drug was also studied as monotherapy in a phase II trial MYRALL in 36 adult patients with relapsed or refractory acute lymphoblastic leukemia. [77] The administration schedule included up to eight weekly doses $(55,70$, or $90 \mathrm{mg} / \mathrm{m}^{2}$ ), followed by up to 24 biweekly doses as maintenance therapy in responding patients. The most common AEs were pyrexia, diarrhea, and nausea. There was one case of grade 3 motor PN at a dose of $90 \mathrm{mg} / \mathrm{m}^{2}$. The ORR was $25.5 \%$ and the duration of response was 1.9 months. The conclusion drawn from the study was inadequate clinical response rate in patients with relapsed or refractory acute lymphoblastic leukemia, and the study was discontinued.

Promising results were obtained in a phase II study STARLYTE to assess the efficacy and safety of SAR3419 in 61 patients with de novo or transformed relapsed/refractory diffuse large B-cell lymphoma who had previously received rituximab-containing immunochemotherapy. [78] SAR3419 was administered in four weekly $55-\mathrm{mg} / \mathrm{m}^{2}$ doses, followed by biweekly doses until disease progression or unacceptable toxicity. The ORR was nearly $44 \%$. The most common non-hematologic AEs included fatigue (30\%), nausea (23\%), and diarrhea (20\%). Grade 3 and 4 AEs were seen in $38 \%$ of patients and mostly involved hepatotoxicity and abdominal pain. Eye disorders were found in $25 \%$ of patients, and ranged from dry eye syndrome to keratitis. All events were transient and reversible. Five patients experienced PN and two experienced optic neuropathy. The latter resolved with a median of 9 days without any study drug dose modifications. Seven patients who were reported with AEs leading to death died because of progressive disease.

A combination therapy with SAR3419 and rituximab was studied in a phase II trial in 52 patients with relapsed or refractory diffuse large B-cell lymphoma. [79] Among fortyfive evaluable patients, the ORR was $31.1 \%$ and the primary objective of an ORR of $40 \%$ was not met. Median PFS, OS, and DOR were 3.9, 9 and 8.6 months, respectively. Common AEs were similar to those reported in previous studies and the pharmacokinetics of the two drugs was unaffected. Current clinical trials are listed in Table 2.

\section{Conclusion}

Currently, there are about 110 ongoing studies implementing ADCs in the treatment of multiple human malignancies. Antibody-drug conjugates are potent with manageable safety issues. The excellent risk-to-benefit ratio warrants further exploration of this treatment modality. Perhaps, the future lies in implementing the ADC strategy early in the patient's treatment. Moreover, modification of the cancer microenvironment might be another promising target for ADCs. The use of immune checkpoint inhibitors conjugated with cytotoxins may elegantly kill two birds with one stone and lead to success in treating patients with malignancies.

\section{Compliance with Ethical Standards}

Funding The study was supported in part by grant funding from the Medical University of Lodz, Poland (No. 503/1-093-01/503-11-004) and by the Foundation for the Development of Diagnostics and Therapy, Warsaw, Poland.

Conflict of interest Anna Wolska-Washer and Tadeusz Robak have no conflicts of interest that are directly relevant to the contents of this study.

Open Access This article is distributed under the terms of the Creative Commons Attribution-NonCommercial 4.0 International License (http://creativecommons.org/licenses/by-nc/4.0/), which permits any noncommercial use, distribution, and reproduction in any medium, provided you give appropriate credit to the original author(s) and the source, provide a link to the Creative Commons license, and indicate if changes were made. 


\section{References}

1. Dan N, Setua S, Kashyap VK, Khan S, Jaggi M, Yallapu MM, Chauhan SC. Antibody-drug conjugates for cancer therapy: chemistry to clinical implications. Pharmaceuticals (Basel). 2018;11(2):E32.

2. Gromek SM, Balunas MJ. Natural products as exquisitely potent cytotoxic payloads for antibody-drug conjugates. Curr Top Med Chem. 2015;14(24):2822-34.

3. Jain N, Smith SW, Ghone S, Tomczuk B. Current ADC linker chemistry. Pharm Res. 2015;32:3526-40.

4. Cianfriglia M. The biology of MDR1-P-glycoprotein (MDR1$\mathrm{Pgp}$ ) in designing functional antibody drug conjugates (ADCs): the experience of gemtuzumab ozogamicin. Ann Ist Super Sanita. 2013;49(2):150-68

5. European Medicines Agency. Mylotarg: summary of product characteristics. http://www.ema.europa.eu/docs/en_GB/docum ent_library/EPAR_-_Product_Information/human/004204/ WC500248702. Accessed 11 Dec 2018.

6. Sievers EL, Larson RA, Stadtmauer EA, Estey E, Löwenberg B, Dombret H, Mylotarg Study Group, et al. Efficacy and safety of gemtuzumab ozogamicin in patients with CD33positive acute myeloid leukemia in first relapse. J Clin Oncol. 2001;19(13):3244-54.

7. Petersdorf SH, Kopecky KJ, Slovak M, Willman C, Nevill T, Brandwein $\mathrm{J}$, et al. A phase 3 study of ozogamicin during induction and postconsolidation therapy in younger patients with acute myeloid leukemia. Blood. 2013;121(24):4854-60.

8. Amadori S, Suciu S, Selleslag D, Aversa F, Gaidano G, Musso $\mathrm{M}$, et al. Gemtuzumab ozogamicin versus best supportive care in older patients with newly diagnosed acute myeloid leukemia unsuitable for intensive chemotherapy: results of the randomized phase III EORTC-GIMEMA AML-19 trial. J Clin Oncol. 2016;34(9):972-9.

9. Amadori S, Suciu S, Selleslag D, Stasi R, Alimena G, Baila L, et al. Randomized trial of two schedules of low-dose gemtuzumab ozogamicin as induction monotherapy for newly diagnosed acute myeloid leukaemia in older patients not considered candidates for intensive chemotherapy: a phase II study of the EORTC and GIMEMA leukaemia groups (AML-19). Br J Haematol. 2010;149(3):376-82.

10. Castaigne S, Pautas C, Terré C, Raffoux E, Bordessoule D, Bastie JN, Acute Leukemia French Association, et al. Effect of gemtuzumab ozogamicin on survival of adult patients with de-novo acute myeloid leukaemia (ALFA-0701): a randomised, open-label, phase 3 study. Lancet. 2012;379(9825):1508-16.

11. Senter PD, Sievers EL. The discovery and development of brentuximab vedotin for use in relapsed Hodgkin lymphoma and systemic anaplastic large cell lymphoma. Nat Biotechnol. 2012;30(7):631-7.

12. Younes A, Bartlett NL, Leonard JP, Kennedy DA, Lynch CM, Sievers EL, et al. Brentuximab vedotin (SGN-35) for relapsed CD30-positive lymphomas. N Engl J Med. 2010;363(19):1812-21.

13. Younes A, Gopal AK, Smith SE, Ansell SM, Rosenblatt JD, Savage KJ, et al. Results of a pivotal phase II study of brentuximab vedotin for patients with relapsed or refractory Hodgkin's lymphoma. J Clin Oncol. 2012;30(18):2183-9.

14. Pro B, Advani R, Brice P, Bartlett NL, Rosenblatt JD, Illidge T, et al. Brentuximab vedotin (SGN-35) in patients with relapsed or refractory systemic anaplastic large-cell lymphoma: results of a phase II study. J Clin Oncol. 2012;30(18):2190-6.

15. Moskowitz CH, Nademanee A, Masszi T, Agura E, Holowiecki $\mathrm{J}$, Abidi MH, et al. Brentuximab vedotin as consolidation therapy after autologous stem-cell transplantation in patients with Hodgkin's lymphoma at risk of relapse or progression (AETHERA): a randomised, double-blind, placebo-controlled, phase 3 trial. Lancet. 2015;385(9980):1853-62.

16. Prince HM, Kim YH, Horwitz SM, Dummer R, Scarisbrick J, Quaglino P, ALCANZA Study Group, et al. Brentuximab vedotin or physician's choice in CD30-positive cutaneous T-cell lymphoma (ALCANZA): an international, open-label, randomised, phase 3, multicentre trial. Lancet. 2017;390(10094):555-66.

17. Connors JM, Jurczak W, Straus DJ, Ansell SM, Kim WS, Gallamini A, et al. Brentuximab vedotin with chemotherapy for stage III or IV Hodgkin's lymphoma. N Engl J Med. 2018;378(4):331-44.

18. European Medicines Agency. Kadcyla: summary of product characteristics. http://www.ema.europa.eu/docs/en_GB/document_ library/EPAR_-_Product_Information/human/002389/WC500 158593.pdf. Accessed 11 Dec 2018.

19. Krop IE, Beeram M, Modi S, Jones SF, Holden SN, Yu W, et al. Phase I study of trastuzumab-DM1, an HER2 antibody-drug conjugate, given every 3 weeks to patients with HER2-positive metastatic breast cancer. J Clin Oncol. 2010;28(16):2698-704.

20. Verma S, Miles D, Gianni L, Krop IE, Welslau M, Baselga J, et al. Trastuzumab emtansine for HER2-positive advanced breast cancer. N Engl J Med. 2012;367(19):1783-91.

21. Hurvitz SA, Dirix L, Kocsis J, Bianchi GV, Lu J, Vinholes J, et al. Phase II randomized study of trastuzumab emtansine versus trastuzumab plus docetaxel in patients with human epidermal growth factor receptor 2-positive metastatic breast cancer. J Clin Oncol. 2013;31(9):1157-63.

22. Krop IE, LoRusso P, Miller KD, Modi S, Yardley D, Rodriguez G, et al. A phase II study of trastuzumab emtansine in patients with human epidermal growth factor receptor 2-positive metastatic breast cancer who were previously treated with trastuzumab, lapatinib, an anthracycline, a taxane, and capecitabine. J Clin Oncol. 2012;30(26):3234-41.

23. Krop IE, Kim SB, González-Martín A, LoRusso PM, Ferrero JM, Smitt M, et al. Trastuzumab emtansine versus treatment of physician's choice for pretreated HER2-positive advanced breast cancer (TH3RESA): a randomised, open-label, phase 3 trial. Lancet Oncol. 2014;15(7):689-99.

24. Kim SB, Wildiers H, Krop IE, Smitt M, Yu R, Lysbet de Haas $\mathrm{S}$, et al. Relationship between tumor biomarkers and efficacy in TH3RESA, a phase III study of trastuzumab emtansine (T-DM1) vs. treatment of physician's choice in previously treated HER2-positive advanced breast cancer. Int J Cancer. 2016;139(10):2336-42.

25. European Medicines Agency. Besponsa: summary of product characteristics. http://www.ema.europa.eu/docs/en_GB/docum ent_library/EPAR_-_Product_Information/human/004119/ WC500231261.pdf. Accessed 11 Dec 2018.

26. DeAngelo DJ, Stock W, Stein AS, Shustov A, Liedtke M, Schiffer $\mathrm{CA}$, et al. Inotuzumab ozogamicin in adults with relapsed or refractory CD22-positive acute lymphoblastic leukemia: a phase 1/2 study. Blood Adv. 2017;1(15):1167-80.

27. Kantarjian HM, DeAngelo DJ, Stelljes M, Martinelli G, Liedtke $\mathrm{M}$, Stock W, et al. Inotuzumab ozogamicin versus standard therapy for acute lymphoblastic leukemia. N Engl J Med. 2016;375(8):740-53.

28. Kantarjian HM, DeAngelo DJ, Advani AS, Stelljes M, Kebriaei $\mathrm{P}$, Cassaday RD, et al. Hepatic adverse event profile of inotuzumab ozogamicin in adult patients with relapsed or refractory acute lymphoblastic leukaemia: results from the open-label, randomised, phase 3 INO-VATE study. Lancet Haematol. 2017;4(8):e387-98.

29. Hedrich WD, Fandy TE, Ashour HM, Wang H, Hassan HE. Antibody-drug conjugates: pharmacokinetic/pharmacodynamic modeling, preclinical characterization, clinical studies, and lessons learned. Clin Pharmacokinet. 2018;57(6):687-703. 
30. Gorovits B, Alley SC, Bilic S, Booth B, Kaur S, Oldfield P, et al. Bioanalysis of antibody-drug conjugates: American Association of Pharmaceutical Scientists Antibody-Drug Conjugate Working Group position paper. Bioanalysis. 2013;5(9):997-1006.

31. Hibma J, Knight B. Population pharmacokinetic modeling of gemtuzumab ozogamicin in adult patients with acute myeloid leukemia. Clin Pharmacokinet. 2018. https://doi.org/10.1007/s4026 2-018-0699-5 (Epub ahead of print).

32. Dowell JA, Korth-Bradley J, Liu H, King SP, Berger MS. Pharmacokinetics of gemtuzumab ozogamicin, an antibodytargeted chemotherapy agent for the treatment of patients with acute myeloid leukemia in first relapse. J Clin Pharmacol. 2001;41(11):1206-14

33. Wolenski FS, Xia CQ, Ma B, Han TH, Shyu WC, Balani SK. CYP suppression in human hepatocytes by monomethyl auristatin $\mathrm{E}$, the payload in brentuximab vedotin (Adcetris ${ }^{\circledR}$ ), is associated with microtubule disruption. Eur J Drug Metab Pharmacokinet. 2018;43(3):347-54.

34. Zhao B, Chen R, O'Connor OA, Gopal AK, Ramchandren R, Goy A, et al. Brentuximab vedotin, an antibody-drug conjugate, in patients with CD30-positive haematologic malignancies and hepatic or renal impairment. Br J Clin Pharmacol. 2016;82(3):696-705.

35. European Medicines Agency. Adcetris: summary of product characteristics. http://www.ema.europa.eu/docs/en_GB/document_ library/EPAR_-_Product_Information/human/002455/WC500 135055. Accessed 11 Dec 2018.

36. Gamis AS, Alonzo TA, Meshinchi S, Sung L, Gerbing RB, Raimondi SC, et al. Gemtuzumab ozogamicin in children and adolescents with de novo acute myeloid leukemia improves event-free survival by reducing relapse risk: results from the randomized phase III Children's Oncology Group trial AAML0531. J Clin Oncol. 2014;32(27):3021-32.

37. Oak E, Bartlett NL. A safety evaluation of brentuximab vedotin for the treatment of Hodgkin lymphoma. Expert Opin Drug Saf. 2016;15(6):875-82.

38. Bartlett NL, Chen R, Fanale MA, Brice P, Gopal A, Smith SE, et al. Retreatment with brentuximab vedotin in patients with CD30-positive hematologic malignancies. J Hematol Oncol. 2014;7:24.

39. DeVita MD, Evens AM, Rosen ST, Greenberger PA, Petrich AM. Multiple successful desensitizations to brentuximab vedotin: a case report and literature review. J Natl Compr Canc Netw. 2014;12(4):465-71.

40. Flerlage JE, Metzger ML, Wu J, Panetta JC. Pharmacokinetics, immunogenicity, and safety of weekly dosing of brentuximab vedotin in pediatric patients with Hodgkin lymphoma. Cancer Chemother Pharmacol. 2016;78(6):1217-23.

41. Carson KR, Newsome SD, Kim EJ, Wagner-Johnston ND, von Geldern G, Moskowitz CH, et al. Progressive multifocal leukoencephalopathy associated with brentuximab vedotin therapy: a report of 5 cases from the Southern Network on Adverse Reactions (SONAR) project. Cancer. 2014;120(16):2464-71.

42. Gandhi MD, Evens AM, Fenske TS, Hamlin P, Coiffier B, Engert A, et al. Pancreatitis in patients treated with brentuximab vedotin: a previously unrecognized serious adverse event. Blood. 2014;123(18):2895-7.

43. Nademanee A, Sureda A, Stiff P, Holowiecki J, Abidi M, Hunder N, et al. Safety analysis of brentuximab vedotin from the phase III AETHERA trial in Hodgkin lymphoma in the posttransplant consolidation setting. Biol Blood Marrow Transpl. 2018;24(11):2354-9. https://doi.org/10.1016/j.bbmt.2018.05.026.

44. Alig SK, Dreyling M, Seppi B, Aulinger B, Witkowski L, Rieger CT. Severe cytokine release syndrome after the first dose of brentuximab vedotin in a patient with relapsed systemic anaplastic large cell lymphoma (sALCL): a case report and review of literature. Eur J Haematol. 2015;94(6):554-7.

45. Shen K, Ma X, Zhu C, Wu X, Jia H. Safety and efficacy of trastuzumab emtansine in advanced human epidermal growth factor receptor 2-positive breast cancer: a meta-analysis. Sci Rep. 2016;16(6):23262. https://doi.org/10.1038/srep23262.

46. Diéras V, Miles D, Verma S, Pegram M, Welslau M, Baselga J, et al. Trastuzumab emtansine versus capecitabine plus lapatinib in patients with previously treated HER2-positive advanced breast cancer (EMILIA): a descriptive analysis of final overall survival results from a randomised, open-label, phase 3 trial. Lancet Oncol. 2017;18(6):732-42.

47. Force J, Saxena R, Schneider BP, Storniolo AM, Sledge GW Jr, Chalasani N, et al. Nodular regenerative hyperplasia after treatment with trastuzumab etansine. J Clin Oncol. 2016;34(3):e9-12.

48. Moilanen T, Jokimäki A, Tenhunen O, Koivunen JP. Trastuzumabinduced cardiotoxicity and its risk factors in real-world setting of breast cancer patients. J Cancer Res Clin Oncol. 2018. https://doi. org/10.1007/s00432-018-2682-9 (Epub ahead of print).

49. National Toxicology Program. NTP monograph: developmental effects and pregnancy outcomes associated with cancer chemotherapy use during pregnancy. NTP Monogr. 2013;2:i-214.

50. Jabbour EJ, DeAngelo DJ, Stelljes M, Stock W, Liedtke M, Gökbuget N, et al. Efficacy and safety analysis by age cohort of inotuzumab ozogamicin in patients with relapsed or refractory acute lymphoblastic leukemia enrolled in INO-VATE. Cancer. 2018;124(8):1722-32.

51. Ricart AD. Antibody-drug conjugates of calicheamicin derivative: gemtuzumab ozogamicin and inotuzumab ozogamicin. Clin Cancer Res. 2011;17(20):6417-27.

52. Han TH, Gopal AK, Ramchandren R, Goy A, Chen R, Matous JV, et al. CYP3A-mediated drug-drug interaction potential and excretion of brentuximab vedotin, an antibody-drug conjugate, in patients with CD30-positive hematologic malignancies. J Clin Pharmacol. 2013;53(8):866-77.

53. Wolenski FS, Xia CQ, Ma B, Han TH, Shyu WC, Balani SK. CYP suppression in human hepatocytes by monomethyl auristatin $\mathrm{E}$, the payload in brentuximab vedotin (Adcetris ${ }^{\circledR}$ ), is associated with microtubule disruption. Eur J Drug Metab Pharmacokinet. 2018;43(3):347-54.

54. Haddley K. Trastuzumab emtansine for the treatment of HER2-positive metastatic breast cancer. Drugs Today (Barc). 2013;49(11):701-15.

55. Davis JA, Rock DA, Wienkers LC, Pearson JT. In vitro characterization of the drug-drug interaction potential of catabolites of antibody-maytansinoid conjugates. Drug Metab Dispos. 2012;40(10):1927-34.

56. Naumovski L, Junutula JR. Glembatumumab vedotin, a conjugate of an anti-glycoprotein non-metastatic melanoma protein $\mathrm{B} \mathrm{mAb}$ and monomethyl auristatin $\mathrm{E}$ for the treatment of melanoma and breast cancer. Curr Opin Mol Ther. 2010;12(2):248-57.

57. Yardley DA, Weaver R, Melisko ME, Saleh MN, Arena FP, Forero A, et al. EMERGE: a randomized phase II study of the antibody-drug conjugate glembatumumab vedotin in advanced glycoprotein NMB-expressing breast cancer. J Clin Oncol. 2015;33(14):1609-19.

58. Tai YT, Mayes PA, Acharya C, Zhong MY, Cea M, Cagnetta A, et al. Novel anti-B-cell maturation antigen antibody-drug conjugate (GSK2857916) selectively induces killing of multiple myeloma. Blood. 2014;123(20):3128-38.

59. Tai YT, Anderson KC. Targeting B-cell maturation antigen in multiple myeloma. Immunotherapy. 2015;7(11):1187-99.

60. Cohen AD, Popat R, Trudel S, Richardson PG, Libby EN III, Lendvai N, et al. First in human study with GSK2857916, an antibody drug conjugated to microtubule-disrupting agent directed against B-cell maturation antigen (BCMA) in patients with 
relapsed/refractory multiple myeloma (MM): results from Study BMA117159 Part 1 dose escalation [abstract]. Annual Meeting of the American Society of Hematology. Blood. 2016;128(Suppl. 1): 1148 .

61. Trudel S, Lendvai N, Popat R, Voorhees PM, Reeves B, Libby EN III, et al. Deep and durable responses in patients (pts) with relapsed/refractory multiple myeloma (MM) treated with monotherapy GSK2857916, an antibody drug conjugate against B-cell maturation antigen (BCMA): preliminary results from part 2 of study BMA117159 [abstract]. Annual Meeting of the American Society of Hematology. Blood. 2017;130(Suppl. 1):741.

62. Goldenberg DM, Cardillo TM, Govindan SV, Rossi EA, Sharkey RM. Trop-2 is a novel target for solid cancer therapy with sacituzumab govitecan (IMMU-132), an antibody-drug conjugate (ADC). Oncotarget. 2015;6(26):22496-512.

63. Tagawa ST, Faltas B, Lam E, Saylor P, Bardia A, Hajdenberg J, Morgans AK, et al. Sacituzumab govitecan (IMMU132) for patients with pretreated metastatic urothelial cancer (UC): interim results [abstract no. 858P]. Ann Oncol. 2017;28(Suppl_5):295-v299.

64. Bardia A, Mayer IA, Diamond JR, Moroose RL, Isakoff SJ, Starodub AN, et al. efficacy and safety of anti-trop-2 antibody drug conjugate sacituzumab govitecan (IMMU-132) in heavily pretreated patients with metastatic triple-negative breast cancer. J Clin Oncol. 2017;35(19):2141-8.

65. Golfier S, Kopitz C, Kahnert A, Heisler I, Schatz CA, StelteLudwig B, et al. Anetumab ravtansine: a novel mesothelin-targeting antibody-drug conjugate cures tumors with heterogeneous target expression favored by bystander effect. Mol Cancer Ther. 2014;13(6):1537-48.

66. Bendell J, Blumenschein G, Zinner R, Hong D, Jones S, Infante $\mathrm{J}$, et al. First-in-human phase I dose escalation study of a novel anti-mesothelin antibody drug conjugate (ADC) BAY 94-9343, in patients with advanced solid tumors [abstract]. Cancer Res. 2013;73(8 Suppl):LB-291.

67. Phase II anetumab ravtansine as 2 nd line treatment for malignant pleural mesothelioma (MPM). ClinicalTrials.gov identifier: NCT02610140.

68. ADC Review. Anetumab ravtansine does not meet primary endpoint in second-line mesothelioma. https://adcreview.com/news/ anetumab-ravtansine-not-meet-primary-endpoint-second-linemesothelioma/. Accessesd 11 Dec 2018.

69. Ogitani Y, Aida T, Hagihara K, Yamaguchi J, Ishii C, Harada $\mathrm{N}$, et al. DS-8201a, a novel HER2-targeting ADC with a novel DNA topoisomerase I inhibitor, demonstrates a promising antitumor efficacy with differentiation from T-DM1. Clin Cancer Res. 2016;22(20):5097-108

70. Iwata TN, Ishii C, Ishida S, Ogitani Y, Wada T, Agatsuma T. A HER2-targeting antibody-drug conjugate, trastuzumab deruxtecan
(DS-8201a), enhances antitumor immunity in a mouse model. Mol Cancer Ther. 2018;17(7):1494-503.

71. Tamura K, Shitara K, Naito Y, Shimomura A, Fujiwara Y. Single agent activity of DS-8201a, a HER2-targeting antibody-drug conjugate, in breast cancer patients previously treated with T-DM1: phase 1 dose escalation. Ann Oncol. 2016;27(Suppl. 6):LBA17.

72. Daiichi Sankyo. Daiichi Sankyo presents long-term phase 1 results of antibody drug conjugate DS-8201 in patients with HER2-expressing breast, gastric and other solid cancers at 2018 American Society of Clinical Oncology (ASCO) Annual Meeting. https://www.daiichisankyo.com/media_investors/media_relations/ press_releases/detail/006863/180601_833_E2.pdf. Accessed 11 Dec 2018.

73. Doi T, Shitara K, Naito Y, Shimomura A, Fujiwara Y, Yonemori K, Shimizu C, Shimoi T, et al. Safety, pharmacokinetics, and antitumour activity of trastuzumab deruxtecan (DS-8201), a HER2targeting antibody-drug conjugate, in patients with advanced breast and gastric or gastro-oesophageal tumours: a phase 1 doseescalation study. Lancet Oncol. 2017;18(11):1512-22.

74. Iwata H, Tamura K, Doi T, Tsurutani J, Modi S, Park H, et al. Trastuzumab deruxtecan (DS-8201a) in subjects with HER2-expressing solid tumors: long-term results of a large phase 1 study with multiple expansion cohorts. J Clin Oncol. 2018;36(Suppl.):2501.

75. Younes A, Kim S, Romaguera J, Copeland A, Farial Sde C, Kwak LW, et al. Phase I multidose-escalation study of the anti-CD19 maytansinoid immunoconjugate SAR3419 administered by intravenous infusion every 3 weeks to patients with relapsed/refractory B-cell lymphoma. J Clin Oncol. 2012;30(22):2776-82.

76. Ribrag V, Dupuis J, Tilly H, Morschhauser F, Laine F, Houot $\mathrm{R}$, et al. A dose-escalation study of SAR3419, an anti-CD19 antibody maytansinoid conjugate, administered by intravenous infusion once weekly in patients with relapsed/refractory B-cell non-Hodgkin lymphoma. Clin Cancer Res. 2014;20(1):213-20.

77. Kantarjian HM, Lioure B, Kim SK, Atallah E, Leguay T, Kelly K, et al. A phase ii study of coltuximab ravtansine (SAR3419) monotherapy in patients with relapsed or refractory acute lymphoblastic leukemia. Clin Lymphoma Myeloma Leuk. 2016;16(3):139-45.

78. Trnĕný M, Verhoef G, Dyer MJ, Ben Yehuda D, Patti C, Canales $\mathrm{M}$, et al. A phase II multicenter study of the anti-CD19 antibody drug conjugate coltuximab ravtansine (SAR3419) in patients with relapsed or refractory diffuse large B-cell lymphoma previously treated with rituximab-based immunotherapy. Haematologica. 2018;103(8):1351-8.

79. Coiffier B, Thieblemont C, de Guibert S, Dupuis J, Ribrag V, Bouabdallah R, et al. A phase II, single-arm, multicentre study of coltuximab ravtansine (SAR3419) and rituximab in patients with relapsed or refractory diffuse large B-cell lymphoma. Br J Haematol. 2016;173(5):722-30. 